\title{
A common tag nucleotide variant in MMP7 promoter increases risk for hypertension via enhanced interactions with CREB transcription factor
}

\author{
by \\ Lakshmi Subramanian ${ }^{1}$, Sakthisree Maghajothi ${ }^{1}$, Mrityunjay Singh², Kousik Kesh ${ }^{3}$, \\ Kalyani Ananthamohan ${ }^{1}$, Saurabh Sharma ${ }^{4}$, Madhu Khullar ${ }^{4}$, Suma M. Victor ${ }^{5}$, \\ Snehasikta Swarnakar ${ }^{3}$, Shailendra Asthana ${ }^{2}$, Ajit S. Mullasari ${ }^{5}$, Nitish R. Mahapatra ${ }^{1}$
}

from the

${ }^{1}$ Department of Biotechnology, Bhupat and Jyoti Mehta School of Biosciences, Indian Institute of Technology Madras, Chennai - 600036, India

2Drug Discovery Research Center, Translational Health Science and Technology Institute, NCR Biotech Science Cluster, Faridabad, Haryana - 121001, India

${ }^{3}$ Drug Development Diagnostic and Biotechnology Division, Indian Institute of Chemical Biology, Kolkata - 700032, India

${ }^{4}$ Department of Experimental Medicine and Biotechnology, Postgraduate Institute of Medical Education and Research, Chandigarh - 160012, India

5Institute of Cardiovascular Diseases, Madras Medical Mission, Chennai - 600037, India

Running title: MMP7 promoter polymorphism and hypertension risk

\section{Address for correspondence:}

Dr. Nitish R. Mahapatra, Department of Biotechnology, Bhupat and Jyoti Mehta School of Biosciences, Indian Institute of Technology Madras, Chennai 600036, India. Tel: 9144-2257-4128; E-mail: nmahapatra@iitm.ac.in 


\section{Abstract}

MMP7 (Matrilysin), a potent extracellular matrix degrading enzyme with wide substrate specificity, is emerging as a new regulator of cardiovascular diseases including coronary artery disease and atherosclerosis. However, potential contributions of MMP7 genetic variations to hypertension remain unknown. In this study, we first probed for the association of a tag single nucleotide polymorphism (SNP) in the MMP7 gene promoter (-181A/G; rs11568818) with hypertension in an urban south Indian population ( $n=1517)$. The heterozygous $A / G$ genotype showed a strong association with hypertension as compared to the $\mathrm{A} / \mathrm{A}$ wild-type genotype $\left(\mathrm{OR}=1.641,95 \% \mathrm{Cl}=1.276-2.109 ; \mathrm{p}=1 \times 10^{-4}\right)$; AG genotype carriers also displayed significantly higher diastolic blood pressure and mean arterial pressure than AA genotype subjects. The study was replicated in a north Indian population $(n=977)$ as well $(O R=1.520,95 \% C l=1.106-2.090 ; p=0.01)$. Transient transfection experiments using MMP7 promoter-luciferase reporter constructs revealed that the variant $-181 \mathrm{G}$ allele conferred greater promoter activity than the $-181 \mathrm{~A}$ allele. Computational prediction and structure-based conformational and molecular dynamics simulation studies suggested higher binding affinity for the transcription factor CREB to the $-181 \mathrm{G}$ promoter. In corroboration, over-expression/down-regulation of CREB and chromatin immunoprecipitation experiments provided convincing evidence for stronger binding of CREB with the $-181 \mathrm{G}$ promoter. Further, the $-181 \mathrm{G}$ promoter also displayed an enhanced response to hypoxia and epinephrine-treatment. The higher promoter activity of $-181 \mathrm{G}$ allele also translated to increased MMP7 protein levels. Indeed, MMP7-181A/G heterozygous individuals displayed elevated plasma MMP7 levels which positively correlated with blood pressure. In conclusion, the MMP7 A-181G promoter SNP increased expression of MMP7 under pathophysiological (such as hypoxic stress and catecholamine excess) conditions via increased interactions with the transcription factor CREB and enhanced the risk for hypertension in its carriers.

Keywords: MMP7, hypertension, single nucleotide polymorphism, association study, transcriptional regulation, CREB, epinephrine, hypoxia. 


\section{INTRODUCTION}

Matrix metalloproteinases (MMPs) are enzymes that degrade the components of extracellular matrix $(\mathrm{ECM})$, basement membrane thereby contributing to tissue remodelling and developmental processes. They are also known to cleave bioactive molecules, chemokines, cytokines, growth factors involved in physiological processes including inflammation, angiogenesis, wound healing etc. ${ }^{1}$ To date, 24 different MMPs have been identified and classified into collagenases, stromelysins, gelatinases, matrilysins and membrane-type MMPs based on their domain and substrate specificities. MMP gene expression is primarily regulated the transcriptional level and their activities are modulated by zymogen activation and specific inhibitors called tissue inhibitors of matrix metalloproteinases and non-specific inhibitors like a2macroglobulin. ${ }^{2}$

MMP7, the smallest (molecular weight: $28 \mathrm{kDa}$ ) matrix metalloproteinase, is a secreted matrilysin with specificity for a broad range of substrates (viz. fibronectin, elastin, type IV collagen and proteoglycans). MMP7 is also known to cleave pro-heparin binding epidermal growth factor, Fas ligand, E-cadherin, insulin-like growth factor binding protein, $\beta 2$ adrenergic receptor, VEGFR2, TNF- $\alpha$ and activate other MMPs like pro-MMP2 and pro-MMP9. ${ }^{3}$ Serum/Plasma MMP7 levels are found to be elevated in several types of cancers including gastric cancer, colorectal cancer, ovarian cancer, lung cancer, prostate cancer etc, atherosclerosis, hypertension, chronic kidney disease and diabetes. ${ }^{4-7}$ Since uncontrolled proteolytic processes leading to vascular remodelling acts as an important determinant of cardiovascular complications including hypertension, myocardial infarction and stroke, MMPs are expected and shown to serve as potential mediators in these disease states. ${ }^{8}$ For instance, knock-down of MMP7 in the spontaneous hypertensive rat model attenuated hypertension. ${ }^{9}$ MMP7 was found to be an upstream transcriptional activator of MMP-2 and knock-down of MMP7 prevented the progression of angiotensin II (Ang II)-induced hypertension and cardiac hypertrophy. ${ }^{10}$ Genetic polymorphisms in the MMP7 have been shown to be associated with coronary artery disease (CAD), acute MI, multiple sclerosis, rheumatoid arthritis, and several cancers. ${ }^{7}, 11$ The MMP7 promoter SNPs A-181G and C-153T, among the 
most widely studied SNPs, were shown to be functional as they displayed allele-specific effects and modulated gene expression due to differential interaction of nuclear binding proteins. ${ }^{11,} 12$ However, potential association of regulatory variants in MMP7 gene promoter with hypertension has not been reported so far. We hypothesize that association studies in ethnically distinct populations and functional characterization of genetic variants in MMP7 promoter may help understand the mechanisms behind dysregulated MMP7 levels in cardiovascular diseases (CVDs) as transcriptional regulation plays a key role in controlling MMP7 gene expression levels.

In this study, we probed for association of the common tag SNP A-181G (rs11568818) in the MMP7 gene promoter using a case-control approach in two geographically distinct Indian populations. The A-181G polymorphism increased risk for hypertension in both study populations. Functional characterization revealed that the variant MMP7-181G promoter displayed higher promoter activity than the wild-type MMP7-181A promoter. Systematic computational, cellular and molecular studies established allele-specific regulation by the transcription factor cAMP response element-binding protein (CREB) in enhancing the MMP7-181G promoter activity in basal as well as in pathophysiological conditions. In corroboration, the carriers of $181 \mathrm{~A} / \mathrm{G}$ heterozygous genotype displayed elevated plasma MMP7 levels and higher blood pressure justifying the functional role of this polymorphism to enhance the risk for hypertension.

\section{MATERIALS AND METHODS}

The detailed methods are provided in the Supplementary Information.

\section{Human Subjects}

The primary study population comprised of 862 hypertensive and 655 normotensive individuals from an urban Chennai population recruited at the Madras Medical Mission (MMM), Chennai. A replication study was carried out with 519 hypertensive and 458 normotensive individuals from Chandigarh recruited at the Postgraduate Institute of Medical Education and Research (PGIMER), Chandigarh. Each volunteer gave an 
informed written consent and the study was approved by the institutional ethics Committee at Indian Institute of Technology Madras. Demographic, physiological and biochemical parameters of both the study populations are listed in the Tables $\mathrm{S} 1$ and S2.

\section{Genotyping of MMP7-181A/G polymorphism}

Genomic DNA was isolated from heparin/EDTA-anticoagulated blood samples using Flexigene DNA kit. The promoter region of MMP7 comprising of $-302 \mathrm{bp}$ to $-153 \mathrm{bp}$ (NCBI accession number: NM_002423.4) was PCR-amplified using specific primers, purified and digested with EcoRI to detect the genotypes at the -181 bp position (Fig. S1).

\section{Estimation of biochemical parameters}

Common biochemical parameters such as glucose, lipid profile, urea, creatinine, hemoglobin, sodium and potassium levels in plasma were estimated by standard assays. Blood pressure was measured in the sitting position using a brachial oscillometric cuff by experienced nursing staff and the readings were recorded in triplicate and averaged. Plasma MMP7 levels were measured by an ELISA kit.

\section{Cloning and mutagenesis}

The MMP7-181G and MMP7-181A constructs were generated in pGL3-Basic vector using specific primers; these constructs harbor -230 bp to +22 bp region of MMP7 gene. MMP7-181A/G promoter-cDNA constructs were generated by replacing the firefly luciferase cDNA in MMP7-181A and MMP7-181G promoter-reporter constructs with MMP7 cDNA.

\section{Cell lines, transfection and reporter assays}

Human neuroblastoma IMR-32, SH-SY5Y, rat cardiomyoblast H9c2, and mouse neuroblastoma N2a cell lines were obtained from the National Center for Cell Sciences, Pune, India. All transfections were carried out using Targefect F2 transfection reagent. MMP7 promoter-reporter constructs and $\beta$-galactosidase ( $\beta$-gal) expression plasmid (internal control) were transfected into these cell lines. Luciferase and $\beta$-galactosidase 
assays were performed as reported previously ${ }^{13}$ after 24-30 hrs of transfection and promoter activities were expressed as luciferase/ $\beta$-galactosidase readings.

Co-transfection experiments with CREB and KCREB expression plasmids ${ }^{14}$, CREB dicer substrate siRNA oligos and treatments (epinephrine and hypoxia) were carried out in IMR-32 and H9c2 cells along/after transfection with MMP7-181G and MMP7-181A promoter-reporter constructs. Luciferase activity and total protein levels were estimated and promoter activities were expressed as luciferase/ $\mathrm{ug}$ of protein. Experiments were also carried out to imitate the homozygous and heterozygous conditions in cella with MMP7 promoter-reporter constructs and by transfecting MMP7 promoter-cDNA constructs to estimate MMP7 levels in vitro.

\section{Western blotting}

Immunoblotting experiments were performed to detect over-expression or downregulation of CREB, phospho-CREB and HIF-1a after transfection experiments/epinephrine treatment/hypoxia and to measure the influence of MMP7$181 \mathrm{G}$ and $-181 \mathrm{~A}$ promoter variants on the MMP7 levels in vitro using specific antibodies.

\section{Chromatin immunoprecipitation (ChIP) assays}

ChIP assays were carried out in N2a and H9c2 cells transfected with MMP7-181G and MMP7-181A promoter-reporter constructs with/without treatment with epinephrine (5 $\mu \mathrm{M}$ ) or hypoxia following our recently reported protocol. ${ }^{5}$ Immunoprecipitation reactions were carried out using CREB/phospho-CREB antibodies with IgG serving as negative control. The region encompassing the MMP7-181A/G promoter was amplified by qPCR using specific primers and the amount of DNA immunoprecipitated in case of $-181 \mathrm{G}$ and -181A alleles due to CREB binding were quantified by the fold enrichment method relative to IgG signal.

\section{Computational analysis}

The homology model of CREB1 was built using MODELLER v11.915, using the template 1DH3 (285-339, Mus musculus, mCREB1, resolution: $2.8 \AA$ ). The MMP7-181G and MMP7-181A promoter DNA models were built using 3DDART ${ }^{16}$ and SCFBio (http://www.scfbio-iitd.res.in/research/drugdna.html). Protein-DNA docking was 
performed with $\mathrm{HDOCK}^{17}$ and $\mathrm{HADDOCK}^{18}$ tools. Molecular dynamics (MD) simulation study for all CREB1-MMP7 (protein-DNA) complexes/models were carried out using Desmond ${ }^{19,} 20$ tool in Maestro.

\section{Data presentation and statistical analysis}

Phenotypic measurements of the study population were expressed as mean \pm SEM. Genotype-phenotype associations were tested by one-way ANOVA with post-hoc tests or Levene's test for equality of variances followed by two-tailed t-test, as appropriate, using Statistical Package for Social Sciences (SPSS). Promoter-reporter transfections results were expressed as mean \pm SEM from representative experiments. Statistical significance was calculated by Student's t-test, one-way ANOVA or two-way ANOVA with post-hoc tests, as applicable, using Prism 5 program.

\section{RESULTS}

Identification and linkage disequilibrium analysis of SNPs in the MMP7 promoter. SNPs occurring at a frequency $\geq 1 \%$ in the $5 \mathrm{~kb} M M P 7$ promoter in the south Asian population of the 1000 Genomes project were identified from dbSNP database (Fig. 1 and Table S3). Of the 10 SNPs identified, 7 were found to be common polymorphisms (occurring at a frequency of $\geq 5 \%$ ). Pair-wise linkage disequilibrium (LD) analysis was carried out to predict the non-random association of the alleles of these ten SNPs in the MMP7 promoter and to shortlist tag SNPs, if any. Eight of the ten SNPs constituted a haplotype block suggesting that these alleles could be inherited together (Fig. 1B). Additionally, tag SNP prediction among these SNPs carried out using LD tag SNP selection tool from SNPinfo ${ }^{21}$ web server predicted the rs11568818 (MMP7 A-181G) polymorphism occurring at a frequency of 0.36 (minor allele frequency (MAF)) in the overall 1000 Genomes population and 0.43 in the south Asian super-population as a tag SNP. The variant at -181 bp was found to be in LD with SNPs at $-1378 \mathrm{bp}$ (rs17098318) and -1773 bp (rs17881620) (Fig. 1B).

\section{Occurrence of MMP7-181 SNP in Indian populations.}


The MMP7 A-181G polymorphism was found to occur at a minor allele frequency (MAF) of 0.473 ( $72 \%$ of the study population), in the Chennai population comprising of 1517 hypertensive and normotensive individuals (Table 1). The study was replicated in 977 hypertensive and normotensive individuals from Chandigarh. The MAF of the MMP7 A$181 \mathrm{G}$ SNP in the Chandigarh population was slightly lesser than that of the Chennai population, although still highly prevalent at 0.412 (64.7\% of the study population). The genotypic frequencies in both populations were found to be in Hardy-Weinberg equilibrium (Chennai population: $X^{2} p=0.99$ and Chandigarh population: $X^{2} p=0.37$ ) (Table 1).

\section{MMP7 -181AG genotype is associated with hypertension in Indian populations.}

Logistic regression analysis was carried out to study the relative risk contributed by the variant $G$ allele towards hypertension after dividing the Chennai and Chandigarh populations into hypertensive cases and normotensive controls by both genotypic (AA vs $A G$ and $A A$ vs $G G$ ) and dominant ( $A A$ vs $A G+G G$ ) models. The -181AG heterozygous genotype was found to be significantly associated with hypertension risk when compared to -181AA genotype with an unadjusted odds ratio (OR) of 1.675 (95\% Confidence Interval $\left.(\mathrm{Cl})=1.315-2.133 ; \mathrm{p}=3.0 \times 10^{-5}\right)$ in Chennai population and 1.505 $(95 \% \mathrm{Cl}=1.136-1.993 ; p=0.004)$ in Chandigarh population (Table 2). The significance of these associations were retained even after adjusting for age, sex and body mass index [BMI] (Chennai population: $\mathrm{OR}=1.641 ; 95 \% \mathrm{Cl}=1.276-2.109 ; \mathrm{p}=1.0 \times 10^{-4}$ and Chandigarh population: $\mathrm{OR}=1.520 ; 95 \% \mathrm{Cl}=1.106-2.090 ; \mathrm{p}=0.01)$. Although the homozygous variant GG genotype did not show a statistically significant odds ratio, the dominant model $(A G+G G)$ exhibited a significantly strong association with hypertension in both Chennai (adjusted $\mathrm{OR}=1.408 ; 95 \% \mathrm{Cl}=1.113-1.781 ; \mathrm{p}=0.004$ ) and Chandigarh (adjusted $\mathrm{OR}=1.464 ; 95 \% \mathrm{Cl}=1.085-1.977 ; \mathrm{p}=0.013$ ) populations (Table 2).

\section{Differential activities of MMP7 -181A/G promoter-reporter constructs under basal conditions.}

To test the functional role of the $-181 \mathrm{~A}$ and $-181 \mathrm{G}$ alleles in contributing to the promoter activity of the MMP7 gene, MMP7 promoter-reporter constructs harboring $A$ and $G$ 
alleles at the site of the polymorphism were generated in pGL3-basic vector. MMP7$181 \mathrm{G}$ and -181A promoter-reporter constructs transfected into cardiomyoblast (H9c2) and neuroblastoma cell lines (IMR-32, SH-SY5Y and N2a) showed differential promoter activities (Fig. 2). MMP7-181G construct consistently displayed higher promoter activity than the $-181 \mathrm{~A}$ construct in both cardiomyoblast H9c2 cell line $(\sim 1.7$ fold; $p<0.01)$ and neuronal cell lines like IMR-32, SH-SY5Y and N2a (IMR-32: 1.4 fold; $\mathrm{p}<0.001$; SHSY5Y: $\sim 1.3$ fold; $\mathrm{p}<0.05$; N2a: 1.5 fold; $\mathrm{p}<0.05)$ suggesting that the variation is functional (Fig. 2).

Computational analyses reveal differential interactions of CREB with MMP7181 A/G promoters.

To probe for the possible differential interaction of transcription factors at $-181 b p$ position that could contribute to the differential activity of $-181 \mathrm{G}$ and $-181 \mathrm{~A}$ alleles, computational prediction of transcription factors was carried out using ConSite, MatInspector and P-Match programs. The transcription factor CREB was predicted to bind to the $-181 \mathrm{G}$ allele with higher affinity by both ConSite and P-Match programs. The Transfac position-weight matrix for CREB with binding scores for $-181 \mathrm{~A}$ and $-181 \mathrm{G}$ alleles are shown in the Fig. S2. Following the computational prediction, further structure-based conformational and molecular dynamics simulation studies were carried out using models of CREB and the MMP7 promoter.

The DP-Bind and PredictProtein tools predicted the region spanning amino acid residues 280-341 to have DNA binding affinity and hence the CREB1 homology model was generated for the region corresponding to residues 280-341 of CREB1. Models of MMP7-181A and MMP7-181G promoters were also generated and docking of the CREB1 structure to the promoter DNA structures followed by molecular dynamics simulations were carried out (Figs. 3A, S3 and Table S4).

Since both complexes were stable till 40 ns in MD simulation, the averaged structure was extracted from the first 40 ns simulation to generate the comparable stable interaction map between MMP7-promoter DNA and CREB1. The interface sites were quantified in terms of residue-wise interaction energy, MM-GBSA (Molecular mechanics, the Generalized Born model and Solvent Accessibility) calculations and the lifetime occupancy of hydrogen bonds. The interaction pattern at the binding site of the 
MMP7-181A and MMP7-181G were quantified in terms of hydrogen bonds and hydrophobic contacts. In case of CREB1-chain A:MMP7-181A complex, N293 and R301 were the two main amino acids that participated in the interactions with the promoter DNA segment. The residue N293 formed total four hydrogen bonds (viz. one with A24 nucleotide at DNA-chain C, two with A25 nucleotide at DNA-chain C and one with T23 nucleotide at DNA-chain D) (Figs. 3B and 3D); the residue R301 formed one hydrogen bond with T22 nucleotide at DNA-chain D. On the other hand, the CREB1chain A:MMP7-181G complex apart from having interactions similar to that of MMP7$181 \mathrm{~A}$ complex, also established three additional hydrogen bonds with residue R289 (two hydrogen bonds with G23 nucleotide at DNA-chain C [i.e., the $-181 \mathrm{G}$ variant nucleotide] and one hydrogen bond with T25 nucleotide at DNA-chain D) (Figs. 3C and 3E).

The 2D-interaction map of the MMP7-181A and MMP7-181G systems reflects the same interaction pattern (Fig. S4). The interaction map displays two regions of interactions for CREB1-chain A, between 22-26 nucleotide on MMP7 DNA:chain C (i.e., -180 to -184 bp of MMP7 promoter) and 21-22 nucleotide on MMP7 DNA:chain D (i.e., 185 to -186 bp of MMP7 promoter). Similarly, CREB1-chain B interacts with 29-31 nucleotide on MMP7 DNA:chain C (i.e., -187 to -189 bp of MMP7 promoter) and 15-18 nucleotide on MMP7 DNA:chain D (i.e., -189 to -192 bp of MMP7 promoter). Despite differences in the van der Waals contacts made by the amino acid residues of CREB1chain A and CREB1-chain B with the MMP7 wild-type and mutated promoters, the direct hydrogen bonds between the MMP7-181G nucleotide and R289 residue (identified as a hot-spot residue in case of CREB1:MMP7-181G complex alone but not in CREB1:MMP7-181A complex) could facilitate the favorable binding of CREB to the MMP7-181G promoter with higher affinity over MMP7-181A promoter.

\section{Experimental evidence for interactions of CREB with MMP7-181A/G promoters.}

In view of the prediction of enhanced interactions of CREB with the MMP7-181G allele (Figs. 3 and S4), experimental validation of differential activation of MMP7-181A/G promoters by CREB was carried out. Over-expression of CREB increased the promoter activity of the MMP7 promoter-reporter constructs in a dose-dependent manner with the $-181 \mathrm{G}$ construct exhibiting significantly higher promoter activity than the $-181 \mathrm{~A}$ construct 
in IMR-32 as well as H9c2 cells (IMR-32: one-way ANOVA F=162.9, p<0.0001; H9c2: one-way ANOVA F=43.3, p<0.0001) (Figs. 4A and 4B). Over-expression of CREB was confirmed by Western blotting. In corroboration, co-transfection of dominant negative KCREB plasmid lead to a highly significant dose-dependent decrease (at least 60\%) in the promoter activity of MMP7-181G construct in both IMR-32 and H9c2 cells (IMR-32: one-way ANOVA F=13.72, p<0.0001; H9c2: one-way ANOVA F=11.45, p<0.001) (Figs. 4C and 4D). The $-181 \mathrm{~A}$ construct did not show any change in promoter activity in response to KCREB co-transfection. Down-regulation studies using CREB siRNA oligo also resulted in $\sim 45 \%$ and $\sim 60 \%$ reduction in promoter activity of $-181 \mathrm{G}$ construct when compared to the negative control oligo in IMR-32 and H9c2 cells respectively (IMR-32: one-way ANOVA F=7.13, $p<0.05$; H9c2: one-way ANOVA F=79.02, $p<0.0001$ ) when compared to the $-181 \mathrm{~A}$ construct (Figs. 5A and 5B). Down-regulation of CREB was confirmed by Western blotting.

Further, chromatin immunoprecipitation (ChIP) assays were carried out to study the interaction and preferential binding of CREB with the MMP7-181G promoter in the context of chromatin. Quantitative PCR for purified, immunoprecipitated chromatin from $\mathrm{N} 2 \mathrm{a}$ and $\mathrm{H} 9 \mathrm{c} 2$ cells transfected with MMP7-181G and -181A promoter constructs revealed that enrichment with -181G allele of the MMP7 promoter was $\sim 1.7-$ and $\sim 1$.8fold higher following immunoprecipitation with CREB when compared with $-181 \mathrm{~A}$ allele $(p<0.05)$ (Figs. 5C and 5D). These results strongly suggest that the higher promoter activity of MMP7-181G construct could be attributed to higher binding affinity of CREB towards the $-181 \mathrm{G}$ allele.

\section{Enhanced response of MMP7-181G promoter to epinephrine: Crucial role for CREB.}

Since elevated levels of catecholamines are associated with hypertension we probed the effect of epinephrine and the concomitant role of CREB, if any, on MMP7-181G and -181A promoter activities. Indeed, epinephrine treatment augmented the promoter activity of MMP7-181G construct in a dose-dependent manner up to $\sim 2.5$-fold and $\sim 2.2-$ fold, respectively, in IMR-32 and H9c2 cells (IMR-32: one-way ANOVA F=22.03, $\mathrm{p}<0.0001$; H9c2: one-way ANOVA $\mathrm{F}=17.10, \mathrm{p}<0.0001$ ) (Figs. $6 \mathrm{~A}$ and $6 \mathrm{~B}$ ); on the other hand, MMP7-181A construct did not show such significant increase in promoter activity. 
Consistently, epinephrine treatment enhanced $\sim 4.4$ - and $\sim 12.4$-fold (when normalized to vinculin) increase in phospho-CREB levels in IMR-32 and H9c2 cells, respectively (Figs. 6C and 6D). Further, to test if CREB mediated the activation of MMP7-181G promoter in response to epinephrine, IMR-32 and H9c2 cells were co-transfected with KCREB and MMP7-181G or -181A promoter-reporter constructs followed by epinephrine treatment. Epinephrine, which augmented the promoter activity of MMP7-181G construct significantly failed to evoke a similar response in the KCREB co-transfected condition (IMR-32: two-way ANOVA genotype effect: $F=56.05, p<0.0001$; treatment effect: $F=15.30, p<0.0001$; H9c2: two-way ANOVA genotype effect: $F=16.50, p<0.001$; treatment effect: $\mathrm{F}=17.58, \mathrm{p}<0.0001)$. The $-181 \mathrm{~A}$ construct did not show any significant difference in promoter activity upon epinephrine treatment either in the presence or absence of KCREB (Figs. 6E and 6F).

In ChIP assays using $\mathrm{N} 2 \mathrm{a}$ and $\mathrm{H} 9 \mathrm{c} 2$ cells treated with epinephrine following transfection of the MMP7 promoter constructs, the $-181 \mathrm{G}$ allele displayed significantly higher fold enrichment with CREB (N2a: 5.0-fold; H9c2: 1.8-fold) and phospho-CREB (N2a: 4 -5-fold; H9c2: 3.5-fold) as compared to the -181A allele (N2a: one-way ANOVA F=223.9, p<0.0001; H9c2: one-way ANOVA F=128.1, p<0.0001) (Figs. 6G and $6 \mathrm{H})$. Thus, the epinephrine induced activation of MMP7-181G promoter activity appears to be strongly mediated by CREB.

\section{Hypoxic stress activates MMP7-181G promoter via CREB.}

Since hypoxia is known to increase blood pressure and is a major contributor to cardiac pathophysiology, the effect of hypoxia on the promoter activity of MMP7-181G and $181 \mathrm{~A}$ constructs and the role of CREB towards the same was studied. IMR-32 and $\mathrm{H} 9 \mathrm{c} 2$ cells were subjected to hypoxia after transfection with MMP7-181G and -181A promoter constructs for $6 \mathrm{hrs}$ with/without CREB and KCREB co-transfection. The basal promoter activity of MMP7-181G promoter was enhanced significantly in response to hypoxia in both IMR-32 and H9c2 cells (IMR-32: 1.7-fold; H9c2: 2.3-fold); however, no significant increase was observed in case of the -181A construct (Figs. 7A and 7B). In the CREB co-transfected condition hypoxia treatment further increased the promoter activity of the MMP7-181G construct ( 5.7-fold in IMR-32 and 7.5-fold in H9c2) when compared to MMP7-181A construct (IMR-32: two-way ANOVA genotype effect: F=39.7, 
$p<0.0001$; treatment effect: $F=267.8, p<0.0001 ;$ H9c2: two-way ANOVA genotype effect: $F=30.97, p<0.0001$; treatment effect: $F=73.01, p<0.0001$ ). Similarly, hypoxia resulted in only a modest increase in promoter activity in the KCREB co-transfected cells. Along with HIF-1 $\alpha$ over-expression (IMR-32: 6.6-fold; H9c2: 7.9-fold), CREB (IMR-32: 1.4fold; H9c2: 1.8-fold), and phospho-CREB (IMR-32: 1.5-fold; H9c2: 1.3-fold) levels were also found to be elevated under hypoxia by Western blot analysis (Figs. $7 \mathrm{C}$ and 7D).

Further, the interaction of CREB and phospho-CREB with the $-181 \mathrm{G}$ and $-181 \mathrm{~A}$ alleles of MMP7 promoter under hypoxia was probed in $\mathrm{N} 2 \mathrm{a}$ and $\mathrm{H} 9 \mathrm{c} 2$ cells transfected with MMP7 promoter-reporter constructs. The fold enrichment for CREB (N2a: 1.5-fold; H9c2: 1.4-fold) and phospho-CREB (N2a: 2.3-fold; H9c2: 1.9-fold) observed in case of $-181 \mathrm{G}$ allele in response to hypoxia was significantly higher (Figs. 7E and 7F) than the $-181 \mathrm{~A}$ allele (N2a: one-way ANOVA $\mathrm{F}=63.7, \mathrm{p}<0.0001$; H9c2: one-way ANOVA $F=28.0, p<0.0001)$.

\section{MMP7-181G promoter results in higher MMP7 levels in vitro and in vivo.}

By generating recombinant plasmids wherein hMMP7 cDNA was placed under the control of MMP7-181G and MMP7-181A promoters we probed whether the differential activities MMP7-181G and $-181 \mathrm{~A}$ result in altered MMP7 levels in vitro (Fig. 8A). These constructs were transfected into N2a (Figs. 8B and 8C) and H9c2 (Figs. 8D and 8E) cells and Western blot analysis of cell lysates revealed significantly higher levels of MMP7 in case of $-181 \mathrm{G}$ promoter-driven MMP7 cDNA (N2a: $~ 1.6$-fold; $p<0.0001$; H9c2: $\sim 1.4$-fold; $p<0.05$ ) as compared to $-181 \mathrm{~A}$ promoter driven MMP7 cDNA suggesting that in the genomic context the MMP7-181G promoter is likely to be more active than the $-181 \mathrm{~A}$ promoter.

Further, plasma MMP7 levels in a section of the study population after stratification into MMP7 promoter genotypes revealed that MMP7-181A/G individuals displayed higher plasma MMP7 levels (Fig. 8F) than $-181 \mathrm{~A} / \mathrm{A}$ and $-181 \mathrm{G} / \mathrm{G}$ individuals (one-way ANOVA $F=3.06, p<0.05$ ). Although plasma MMP7 levels were modestly higher in individuals of $-181 \mathrm{G} / \mathrm{G}$ genotype as compared to those with $-181 \mathrm{~A} / \mathrm{A}$ genotype, the difference was not statistically significant. Thus, the plasma levels of MMP7 follow 
the same trend of association displayed by the $-181 \mathrm{~A} / \mathrm{G}$ individuals in the logistic regression analysis towards hypertension risk (Table 2 ).

\section{Activities of MMP7 promoter constructs in diploid combinations in cella.}

In view of the association of MMP7-181A/G genotype with hypertension risk as well as higher MMP7 levels in vivo we sought to test whether this observation could also be demonstrated in transfected cultured cells. We transfected IMR-32 and H9c2 cells with $181 \mathrm{G}$ or $-181 \mathrm{~A}$ promoter-reporter plasmids in three diploid combinations: $-181 \mathrm{G} / \mathrm{G}$ (i.e., only $-181 \mathrm{G}$ construct to mimic the homozygous variant), $-181 \mathrm{G} / \mathrm{A}$ (i.e., equimolar amounts of $-181 \mathrm{G}$ and $-181 \mathrm{~A}$ constructs to mimic the heterozygous condition) and $181 \mathrm{~A} / \mathrm{A}$ (i.e., only -181A construct to mimic the wild-type condition). Interestingly, both the $-181 \mathrm{G}$ and the $-181 \mathrm{G} / \mathrm{A}$ transfected conditions resulted in almost similar extent of elevated promoter activity ( 1.8-fold and $\sim 1.75$-fold, respectively, in IMR-32 cells; 1.4 fold and $~ 1.3$-fold, respectively, in H9c2 cells) than the -181A construct alone (IMR-32: one-way ANOVA F=63.74, $\mathrm{p}<0.0001$; and H9c2: one-way ANOVA F=51.37, $\mathrm{p}<0.001$ ) (Figs. $8 \mathrm{G}$ and $8 \mathrm{H}$ ). These results suggest that the MMP7-181G allele may act in a dominant manner while impacting the promoter activity.

\section{Association of MMP7 -181AG genotype with blood pressure.}

To understand the potential associations of these genotypes with traits influencing cardiovascular physiology inferential statistics was carried out using the phenotypic data for demographic/physiological/biochemical parameters of the study population after stratifying them on the basis of their MMP7-181 promoter genotype. We observed higher mean arterial pressure [MAP] ( 2-3 $\mathrm{mm} \mathrm{Hg})$ and diastolic blood pressure [DBP] $(\sim 2-3 \mathrm{~mm} \mathrm{Hg})$ in individuals of MMP7 -181AG genotype than -181AA or -181GG individuals in both Chennai (MAP: one-way ANOVA F=3.17, $p<0.05$; DBP: one-way ANOVA $F=3.70, p<0.05$ ) and Chandigarh (MAP: one-way ANOVA $F=3.06, p<0.05$; DBP: one-way ANOVA F=3.29, p<0.05) populations (Figs. 9A and 9B). The MAP and DBP levels in these subjects positively correlated with plasma MMP7 levels (MAP: Pearson $r=0.21, p<0.01$; DBP: Pearson $r=0.29$, $p<0.001$ ) (Figs. 9C and 9D). MMP7$181 \mathrm{~A} / \mathrm{G}$ individuals also displayed a trend towards higher systolic blood pressure ( 3 $\mathrm{mm} \mathrm{Hg}$ ), although the difference was not statistically significant (Fig. S5). However, a 
modest positive correlation was observed with plasma MMP7 levels in these individuals (Pearson $r=0.17, p<0.05$ ) (Fig. S5).

\section{DISCUSSION}

\section{MMPs and their role in cardiovascular diseases.}

Hypertension, a major risk factor for cardiovascular disease is characterized by several complex pathophysiological mechanisms including cardiac remodelling. ${ }^{22}$ The remodelling which occurs initially as an adaptive response results in increased/altered ECM content ultimately leading to cardiovascular dysfunction. ${ }^{23}$ MMPs play a crucial role in cardiac remodelling by degrading extracellular matrix components which provide structural and mechanical support to the vasculature. ${ }^{24}$ An imbalance between the activities and levels of MMPs and their inhibitors (TIMPs) has been implicated in the pathogenesis of hypertension and cardiovascular diseases. ${ }^{25}$ Elevated MMP levels have been shown to increase blood pressure via cleavage of pharmacological receptors (e.g., $\beta 2$-adrenergic receptor, epidermal growth factor receptor) and vasoactive peptides (e.g., endothelin, adrenomedullin). ${ }^{8}$

MMP7, the smallest known member of the MMP family is known to cleave a wide range of substrates including ECM components, vasoactive ligands, growth factor receptors, pro-inflammatory molecules and other MMPs. ${ }^{26}$ Knock-down of MMP7 in spontaneous hypertension rat model attenuated hypertension and stopped the development of cardiac hypertrophy. ${ }^{9}$ MMP7 was also shown to be involved in early stages of agonist induced hypertension where it served as a transcriptional regulator of MMP2 in Ang-II induced hypertension. The study revealed that inhibition of MMP2 prevented only blood pressure elevation whereas knock-down of MMP7 and TACE (TNF- $\alpha$ converting enzyme) prevented hypertension as well as the development of cardiac hypertrophy. ${ }^{10}$ Since expression of MMPs including MMP7 is known to be tightly regulated at transcriptional level, polymorphisms in the promoter region may affect the gene expression by altering the binding affinities of transcription factors binding to the transcription factor binding sites in the promoter. Such functional polymorphisms could potentially increase/decrease susceptibility or genetic predisposition to 
pathophysiological phenotypes. ${ }^{11}$ Two polymorphisms in the MMP7 promoter (rs11568818:A-181G and rs11568819:C-153T) have been shown to be associated with the risk of different types of cancers, coronary artery disease ${ }^{7,} 11$ but no studies have reported a direct association of these variants with hypertension risk. Hence, we sought out to study the association of MMP7 promoter variation with hypertension risk if any in two geographically distinct Indian populations using a systematic case-control approach followed by functional characterization of the associated variation.

Occurrence and association of MMP7A-181G polymorphism with hypertension in geographically distinct Indian populations.

We first performed pair-wise linkage disequilibrium analysis of common SNPs in the MMP7 upstream promoter region (Fig. 1) and identified rs11568818 (MMP7 A-181G) as a tag SNP, which was used for further genotyping and functional characterization. The MMP7 A-181G polymorphism was found to occur at a frequency of 0.473 in the primary Chennai population and at a frequency of about 0.412 in the replication population from Chandigarh (Table 1). The polymorphism occurred at a frequency similar to that observed in the European, south Asian and African super populations (EUR: 0.44, SAS: 0.43, AFR: 0.45) of the 1000 Genomes project. Interestingly, the polymorphism occurred at a lesser frequency of 0.34 in the American super population and was least frequent in the east Asian super population with a mere frequency of 0.085 suggesting an ethnicity dependent occurrence of the SNP in different populations.

Next, we probed for association of the MMP7 A-181G polymorphism with hypertension risk. The $-181 \mathrm{~A} / \mathrm{G}$ genotype conferred at least $\sim 1.5$-fold higher risk of hypertension in both the Chennai population and in the replication population from Chandigarh with the dominant model also displaying a highly significant association with hypertension risk (Table 2). A multitude of studies have demonstrated the association MMP7 A-181G polymorphism with several types of cancers, however only limited reports are available characterizing the association of this SNP in cardiovascular disorders. In a meta-analysis of 25 studies comprising of 6392 cases and 7665 controls that suggested a higher overall cancer risk for the MMP7 A-181G polymorphism especially among Asian populations, the GG genotype was particularly associated with 
increased risk of colorectal cancer. ${ }^{27}$ The heterozygous $A / G$ genotype also enhanced susceptibility to grade II astrocytoma by 2 -fold while $G / G$ genotype increased susceptibility of grade II to IV astrocytoma by 3 -folds. ${ }^{28}$ The MMP7 $-181 \mathrm{G}$ allele was also found to significantly increase the risk of esophageal carcinoma, gastric cardiac adenocarcinoma, non-small cell lung cancer, ovarian cancer. ${ }^{7,29}$ In a study involving a north Indian population, an increased risk of cervical cancer was conferred on to individuals with $-181 \mathrm{GG}$ genotype (OR=1.94) or $-181 \mathrm{G}$ allele $(\mathrm{OR}=1.37) .{ }^{30}$ Recently, we showed association of MMP7 -181GG genotype with increased gastric cancer susceptibility $(\mathrm{OR}=1.9 ; \mathrm{p}=0.02) .{ }^{5}$ Although no independent association of MMP7 A$181 \mathrm{G}$ polymorphism was observed with left ventricular dysfunction in CAD patients in a study conducted in north Indian population ${ }^{31}$, high order gene-gene interaction analysis using classification and regression tree (CART) and multifactor dimensionality reduction (MDR) with four-factor interaction model predicted that individuals carrying the combination of AT1 1166AC+CC, NFKB1 -94 ATTG Ins/Ins, MMP7 -181AG+GG, and MMP9 668RQ+QQ genotypes displayed a significantly higher risk for LVD (adjusted $\mathrm{OR}=8.14 ; \mathrm{p}=0.003) .{ }^{32}$ To follow up this first report on the association of MMP7 A-181G SNP with hypertension risk it would be interesting to carry out systematic large-scale studies on the potential association of this genetic variation with related cardiovascular disease states in different human populations.

\section{Allele-specific effect of CREB in activation of MMP7-181G promoter in basal and pathophysiological conditions.}

Transient transfections of MMP7-181A and $-181 \mathrm{G}$ promoters revealed that the $-181 \mathrm{G}$ promoter consistently displayed higher promoter activity than the $-181 \mathrm{~A}$ promoter across different cell lines suggesting that the variation was functional (Fig. 2). Next, we probed the molecular mechanism behind the observed higher promoter activity of the $181 \mathrm{G}$ promoter. Detailed computational analyses including structure-based conformational and molecular dynamics simulation studies affirmed stronger interaction of CREB transcription factor with the $-181 \mathrm{G}$ promoter (Figs. 3 and S4). Co-transfection experiments in $\mathrm{H} 9 \mathrm{c} 2$ and IMR-32 cells with CREB over-expression, dominant negative constructs and siRNA mediated down-regulation studies corroborated the results from the computational analysis; the $-181 \mathrm{G}$ promoter construct displayed a higher promoter 
activity in response to CREB over-expression (Figs. 4A and 4B) while exhibiting a greater reduction in promoter activity in response to CREB knock-down experiments (Figs. 4 and 5) as compared to the $-181 \mathrm{~A}$ promoter construct. Chromatin immunoprecipitation studies further confirmed enhanced interaction of CREB with $181 \mathrm{G}$ promoter as compared to $-181 \mathrm{~A}$ promoter (Fig. 5) proving the mechanistic basis for the higher activity of the $-181 \mathrm{G}$ promoter.

CREB is a ubiquitously expressed transcription factor belonging to the leucine zipper class of transcription factors with well-known roles in cell proliferation, differentiation, survival and known to be activated by various extracellular stimuli. CREB was initially identified from studies on the cAMP dependent activation of somatostatin gene promoter where it was found to bind an 8 bp cAMP-responsive element (CRE), 5'TGACGTCA-3'. 33 Several kinases including protein kinase A (PKA), protein kinase B (AKT), protein kinase C (PKC), MAP kinase activated protein kinase 2 (MAPKAP-2), Ca2+/calmodulin dependent protein kinases II and IV have been shown to activate CREB by phosphorylating the Ser-133 residue which in turn recruits the CREB binding protein (CBP) to activate gene expression. ${ }^{34}$ Targets of CREB include many metabolic enzymes (e.g., Hydroxymethylglutaryl-CoA [HMG CoA] synthase, phosphoenolpyruvate carboxykinase [PEPCK], glucose 6-phosphatase [G6Pase]), neurotransmitters (e.g., Glucagon, tyrosine hydroxylase, chromogranins A and B, enkephalin, vasopressin), transcription factors (e.g., NF-IL-6, Epidermal growth factor-1, STAT-3, c-Fos, PGC-1), growth factors (e.g., Insulin, TNF- $\alpha$, brain derived neutrophic factor [BDNF], cardiotrophin), cell cycle and survival related proteins (e.g., Retinoblastoma, cyclin A, cyclin D1, Bcl-2), immune regulatory proteins (e.g., Interleukin-2, Interleukin-6, Cox-2) and structural proteins (e.g., fibronectin, $\alpha$ A-crystallin). ${ }^{34}$

Although CREB is known to play an important role in neuropsychiatric disorders, its role in the development of cardiovascular diseases has been recently recognized. 35 Over-expression of cardiomyocyte-specific dominant negative CREB in transgenic mice under the influence of a-myosin heavy chain gene promoter reduced the cardiac contractility in response to isoproterenol stimulation mirroring idiopathic dilated cardiomyopathy. ${ }^{36}$ Phospho-CREB levels were also found to be elevated in cerebral 
arteries of hypertensive rats. ${ }^{37}$ Phospho-CREB levels also correlated with the proliferative response resulting in arteriolar injury in Ang II induced hypertension and endothelial injury. ${ }^{38}$ Involvement of CREB in Ang II induced IL-6 expression in vascular smooth muscle cells was attributed to a crucial CRE site. Ang II activated several kinases via AT1-receptor to result in CREB phosphorylation suggesting that CREB may have a role to play in the vascular remodeling associated with cardiac hypertrophy, heart failure and atherosclerosis. ${ }^{39}$

Previous reports have indicated that the MMP7 promoter, like most MMP promoters, harbors binding sites for activator protein (AP-1) and polyoma enhancer $A$ binding protein-3 (PEA-3) which in turn interact with the Fos and Jun family and Ets family of transcription factors to activate gene transcription. ${ }^{40,} 41$ MMP7 promoter has also been shown to harbor TGF inhibitory elements (TIEs) which are known to bind the SMAD family of transcription factors. ${ }^{42}$ Wnt signaling is also known to regulate MMP7 by binding $\beta$-catenin and its partners Tcf/Lef-1.43 In the context of the MMP7 A-181G polymorphism, the higher promoter activity of MMP7-181G allele was attributed to the generation of a putative binding site (NGAAN) for a heat-shock transcription factor binding site in case of the $-181 \mathrm{G}$ allele. ${ }^{11}$ Another study investigating the role of MMP7 promoter polymorphisms in idiopathic pulmonary fibrosis, wherein the AA genotype was associated with higher plasma levels, reported the interaction of forkhead box A2 (FOXA2) to the $-181 \mathrm{~A}$ allele of the MMP7 promoter using transient transfection assays and DNA-protein binding assays. ${ }^{12}$ In our earlier study on the association of gastric cancer risk with MMP7 A-181G polymorphism we had also showed that in the gastric adenocarcinoma cell line, AGS, the higher promoter activity of MMP7-181G allele could be attributed to increased binding affinity of CREB to the MMP7-181G promoter. ${ }^{5}$ Thus, the current study by virtue of establishing the CREB-mediated activation of MMP7-181G promoter in cardiomyoblast and neuronal cells as well sheds light on an important role for CREB in the transcriptional regulation of MMP7, especially in cardiovascular diseases.

Since stress elevates catecholamine levels through the hypothalamic-pituitaryadrenal axis $^{44}$ and hypertension is characterized by elevated levels of vasoconstrictive 
agonists such as catecholamines, we checked if epinephrine, a catecholamine and known activator of CREB exhibited any allele specific effects with respect to the MMP7$181 \mathrm{~A} / \mathrm{G}$ promoters. Indeed, the MMP7-181G construct displayed a dose-dependent increase in promoter activity in response to increasing doses of epinephrine. The increase in phospho-CREB levels in response to epinephrine treatment and the failure of epinephrine to augment the promoter activity in KCREB co-transfected cells establishes the concomitant role of CREB in epinephrine-mediated elevation in MMP7$181 \mathrm{G}$ promoter activity (Fig. 6). In the context of chromatin as well, ChIP experiments demonstrated higher promoter occupancy of both CREB and phospho-CREB in case of MMP7-181G promoter in the epinephrine treated condition (Fig. 6). Thus, hypertensive carriers of the $-181 \mathrm{G}$ allele may have even higher expression of MMP7 gene due to abundance of vasoconstrictive agonists like catecholamines. Interestingly, isoproterenol stimulation was found to activate MMP7 expression at mRNA and protein level in gastric cancer cells by inducing AP-1 and STAT3. ${ }^{45}$ Further, increased expression of MMP7 in gastric cancer tissue was observed at the sites where $\beta 2$-adrenergic receptor was overexpressed emphasizing the role of epinephrine in activation of MMP7-181G promoter in hypertension and other stress induced cardiac complications.

Similarly, hypoxia, a condition resulting in diminished oxygen supply to the tissues is often a pathophysiological occurrence in cardiovascular diseases. HIF-1 $\alpha, \mathrm{HIF}-2 \alpha$ are the most commonly encountered mediators of hypoxia. ${ }^{46} \mathrm{HIF}-1 \alpha$ activity is regulated at post-translational level wherein under normoxic conditions it undergoes proteasomal degradation while under hypoxic conditions the protein is stabilized and translocated to the nucleus allowing it to bind to the hypoxia responsive elements and activating gene transcription. ${ }^{47,48}$ Hypoxia, in fact, is a common pathophysiological feature in CVDs including atherosclerosis and heart failure. Normotensive Sprague-Dawley rats developed sustained arterial hypertension when subjected to hypobaric hypoxia. ${ }^{49}$ In SHR model of hypertension, the brainstem was found to be hypoxic which lead to increased release of ATP and lactate resulting in elevated sympathetic activity and a corresponding increase in arterial blood pressure. ${ }^{50}$ Chronic hypoxia is often found to be associated with increased sympathetic activity and systemic arterial hypertension. In a study subjecting Danish lowlanders at sea level to an altitude of $5260 \mathrm{~m}$ for 9 weeks, 
the mean blood pressure was found to be $28 \%$ higher with up to a $41 \%$ increase in DBP accompanied by 3.7- and 2.4- fold higher plasma arterial norepinephrine and epinephrine levels. ${ }^{51} \mathrm{HIF}-1 \alpha$ signaling has been identified to play important roles in regulation of inflammation (e.g., in macrophage activation) which in turn contributes to the tissue remodeling processes thereby influencing the severity of CVDs. ${ }^{52}$

Although the hypoxic response is primarily mediated by HIF-1a, reports have also shown the involvement of NF-kB and CREB transcription factors. The DNA binding domain of CREB harbors two Cys residues (at positions 300 and 310) which were found to be crucial for the binding of CREB to the CRE site. Mutational analysis revealed that these residues may also act as oxygen sensors resulting in increased expression of CREB-mediated genes in response to hypoxia. ${ }^{53}$ Electrophoretic mobility shift experiments revealed that CREB and ATF-1 were capable of binding to the HIF-1 DNA recognition site. ${ }^{54}$ CREB was further shown to be up-regulated along with ATF-1 and other CREB-responsive genes in response to hypoxia in the lung tissue of mice in vivo. ${ }^{55}$ In PC12 cells, hypoxia resulted in Ser-133 phosphorylation of CREB which in turn activated tyrosine hydroxylase. Interestingly, the phosphorylation of CREB observed in response to hypoxia was maximal with a 3-6 hrs exposure and continued to persist for at least 24 hours. ${ }^{56}$ PKA mediated CREB activation was also observed in the ischemic heart. ${ }^{57}$

In line with these reports, hypoxia significantly enhanced the promoter activity of MMP7-181G construct under basal as well as CREB co-transfected conditions when compared to cells maintained in normoxia. However, no significant increase in response to hypoxia was observed when the cells were co-transfected with the dominant negative KCREB construct suggesting that the increased promoter activity of MMP7-181G construct under hypoxia is modulated by CREB. ChIP experiments revealing increased promoter occupancy of CREB under hypoxic condition in case of the MMP7-181G promoter and elevated levels of CREB and phospho-CREB in response to hypoxia further corroborated our findings to confirm the role of CREB in mediating hypoxia induced activation of MMP7-181G promoter (Fig. 7). Corroborating our findings, primary human monocyte-derived macrophages subjected to $16 \mathrm{hrs}$ of hypoxia displayed 
elevated mRNA levels of MMP7 apart from that of VEGF and GLUT-1. Hypoxia also caused an increase in the promoter activity of MMP-7 and GLUT-1 as shown through reporter assays in RAW 264.7 macrophages indicating an activation of gene expression at transcriptional level. MMP7 mRNA and protein levels were shown to be up-regulated in hypoxic macrophages in vitro and in vivo in hypoxic areas of breast tumors. ${ }^{58}$ Thus, the MMP7 A-181G polymorphism has a functional role in governing gene expression under not only basal condition but also in response to pathophysiological stimuli.

\section{Genotype-phenotype correlations of MMP7 A-181G polymorphism.}

We observed that expression of MMP7 under the influence of MMP7-181G promoter was significantly higher than that of MMP7-181A promoter suggesting that in the genomic context, the higher promoter activity of the MMP7 A-181G SNP translated to higher protein levels in vitro (Figs. 8 A-E). Plasma MMP7 levels, though modest, were significantly higher in individuals of $A / G$ genotype when compared to wild-type $A / A$ individuals (Fig. 8F) while the plasma MMP7 levels in individuals of variant $G / G$ genotype although higher when compared to wild-type A/A individuals was not statistically significant. Since the heterozygous genotype showed a strong association with hypertension risk in our study populations as well as significantly higher plasma MMP7 levels, we transfected diploid combinations of $-181 \mathrm{~A} /-181 \mathrm{G}$ constructs mimicking the homozygous and heterozygous combinations in cella to study the effect of heterozygous genotype on MMP7 transcription. Interestingly, the $-181 \mathrm{~A} / \mathrm{G}$ heterozygous combination also yielded significantly higher promoter activity than the $-181 \mathrm{~A}$ wild-type indicating that MMP7 gene expression is likely to be enhanced in the heterozygous individuals as well (Figs. $8 \mathrm{G}$ and $8 \mathrm{H}$ ). Several reports have suggested that altered expression of MMPs in different individuals could result due to the polymorphisms in the regulatory regions (promoter) of MMP genes in these individuals. ${ }^{2}$ Contrary to our finding, the plasma MMP7 levels were higher in individuals of -181AA genotype in idiopathic pulmonary fibrosis (IPF); however, these observations were limited to IPF patients and not in healthy control suggesting that this may be a specific pathological response. ${ }^{12}$ 
Of note, in a preliminary study, hypercholesterolemic patients with CAD possessing the $-181 \mathrm{G}$ allele presented with smaller reference luminal diameters before percutaneous transluminal coronary angioplasty than patients with the wild-type allele suggesting a functional role for the MMP7 A-181G polymorphism in the matrix remodeling associated with CAD. ${ }^{11}$ We also probed for association of the MMP7 A$181 \mathrm{G}$ polymorphism with other physiological/biochemical parameters like total cholesterol, triglycerides, LDL- and HDL-cholesterol and blood glucose levels that may act as co-morbidities to cardiovascular disease; however, no significant correlations were observed. Nonetheless, the significantly higher diastolic blood pressure and mean arterial pressure observed in individuals of $A / G$ genotype (Fig. 9) corroborated with the results from the logistic regression analysis for predicting hypertension risk suggesting plausible contribution for the MMP7 A-181G polymorphism to hypertension.

In summary, we genotyped a common naturally-occurring polymorphism (A-181G) in the upstream regulatory region of MMP7 gene in two geographically distinct Indian populations comprising of 2500 hypertensive and normotensive subjects using a systematic case-control approach. The MMP7 A-181G polymorphism showed strong association with increased hypertension risk in our study populations. The $-181 \mathrm{G}$ allele containing promoter displayed higher promoter activity than the $-181 \mathrm{~A}$ promoter in basal as well as under pathophysiological stimuli (hypoxia, catecholamine excess) due to preferential binding with CREB transcription factor which translated to increased MMP7 levels in vitro (Fig. 10). The risk genotype (-181A/G) also had a correlative association with increased plasma MMP7 levels and blood pressure suggesting that this functional regulatory polymorphism may contribute to cardiovascular risk.

\section{Perspectives.}

Uncontrolled proteolytic processes modulated by MMPs resulting in cardiac remodeling plays a major role in the pathological mechanisms involved in hypertension and the resultant cardiovascular complications. MMP7, a potent metalloproteinase with a wide range of ECM and non-ECM substrates majorly implicated in several cancers, atherosclerosis etc. is, therefore, a logical regulator of cardiovascular mortality. This 
study sheds light on the association of a highly frequent tag SNP in the gene expression controlling promoter region of MMP7 (A-181G; rs11568818) with increased blood pressure and higher risk for hypertension in Indian population; this work also provides a plausible transcription regulatory mechanism behind the observed elevated levels of MMP7 in the carriers of the variation. Thus, the study paves way towards a better understanding of the role of inter-individual variations and functional regulatory polymorphisms in conferring disease risk, which may be used for developing preventive strategies in individuals likely to be predisposed to hypertension and the related cardiovascular complications.

\section{Acknowledgments}

We acknowledge all the volunteers who participated in this study. We thank Dr. David Ginty (Howard Hughes Medical Institute) for providing the CREB expression plasmid (VP16-CREB+bZIP) and Dr. Richard H. Goodman (Vollum Institute, Oregon Health Sciences University, Portland, OR) for the CREB dominant negative (K-CREB) plasmid.

L. S. acknowledges the clinical research team at MMM, Chennai, for helping with the sample collection and Mr. Abrar Ali Khan, Mr. Vikas Arige and Ms. Amrita Anand for their support during the study.

\section{Sources of funding.}

This study was supported in part by grants from the Department of Biotechnology and Department of Science \& Technology, Government of India to NRM.

Conflicts of interest: None 


\section{REFERENCES}

1. Loffek S, Schilling O, Franzke CW. Biological role of matrix metalloproteinases: A critical balance. Eur Respir J. 2011; 38:191-208

2. Ye S. Polymorphism in matrix metalloproteinase gene promoters: Implication in regulation of gene expression and susceptibility of various diseases. Matrix Biol. 2000; 19:623-629

3. Chiao YA, Zamilpa R, Lopez EF, Dai Q, Escobar GP, Hakala K, Weintraub ST, Lindsey ML. In vivo matrix metalloproteinase-7 substrates identified in the left ventricle post-myocardial infarction using proteomics. J Proteome Res. 2010; 9:2649-2657

4. Ban CR, Twigg SM, Franjic B, Brooks BA, Celermajer D, Yue DK, McLennan SV. Serum MMP-7 is increased in diabetic renal disease and diabetic diastolic dysfunction. Diabetes Res Clin Pract. 2010; 87:335-341

5. Kesh K, Subramanian L, Ghosh N, Gupta V, Gupta A, Bhattacharya S, Mahapatra NR, Swarnakar S. Association of MMP7 -181a-->G promoter polymorphism with gastric cancer risk: Influence of nicotine in differential allelespecific transcription via increased phosphorylation of cAMP-response elementbinding protein (CREB). J Biol Chem. 2015; 290:14391-14406

6. Nilsson L, Jonasson L, Nijm J, Hamsten A, Eriksson P. Increased plasma concentration of matrix metalloproteinase-7 in patients with coronary artery disease. Clin Chem. 2006; 52:1522-1527

7. Zhang J, Jin X, Fang S, Wang R, Li Y, Wang N, Guo W, Wang Y, Wen D, Wei L, Dong $Z$, Kuang $G$. The functional polymorphism in the matrix metalloproteinase-7 promoter increases susceptibility to esophageal squamous cell carcinoma, gastric cardiac adenocarcinoma and non-small cell lung carcinoma. Carcinogenesis. 2005; 26:1748-1753

8. Schmid-Schonbein GW. An emerging role of degrading proteinases in hypertension and the metabolic syndrome: Autodigestion and receptor cleavage. Curr Hypertens Rep. 2012; 14:88-96

9. Wang X, Chow FL, Oka T, Hao L, Lopez-Campistrous A, Kelly S, Cooper S, Odenbach J, Finegan BA, Schulz R, Kassiri Z, Lopaschuk GD, Fernandez-Patron 
C. Matrix metalloproteinase-7 and ADAM-12 (a disintegrin and metalloproteinase-12) define a signaling axis in agonist-induced hypertension and cardiac hypertrophy. Circulation. 2009; 119:2480-2489

10. Odenbach J, Wang X, Cooper S, Chow FL, Oka T, Lopaschuk G, Kassiri Z, Fernandez-Patron C. MMP-2 mediates angiotensin II-induced hypertension under the transcriptional control of MMP-7 and tace. Hypertension. 2011; 57:123130

11. Jormsjo S, Whatling C, Walter DH, Zeiher AM, Hamsten A, Eriksson P. Allelespecific regulation of matrix metalloproteinase-7 promoter activity is associated with coronary artery luminal dimensions among hypercholesterolemic patients. Arterioscler Thromb Vasc Biol. 2001; 21:1834-1839

12. Richards TJ, Park C, Chen Y, Gibson KF, Peter Di Y, Pardo A, Watkins SC, Choi AM, Selman M, Pilewski J, Kaminski N, Zhang Y. Allele-specific transactivation of matrix metalloproteinase 7 by FOXA2 and correlation with plasma levels in idiopathic pulmonary fibrosis. Am J Physiol Lung Cell Mol Physiol. 2012; 302:L746-754

13. Sonawane PJ, Sahu BS, Sasi BK, Geedi P, Lenka G, Mahapatra NR. Functional promoter polymorphisms govern differential expression of HMG-CoA reductase gene in mouse models of essential hypertension. PLoS One. 2011; 6:e16661

14. Riccio A, Ahn S, Davenport CM, Blendy JA, Ginty DD. Mediation by a CREB family transcription factor of NGF-dependent survival of sympathetic neurons. Science. 1999; 286:2358-2361

15. Webb B, Sali A. Protein structure modeling with MODELLER. Methods Mol Biol. 2014; 1137:1-15

16. van Dijk M, Bonvin AM. 3d-dart: A DNA structure modelling server. Nucleic Acids Res. 2009; 37:W235-239

17. Yan Y, Zhang D, Zhou P, Li B, Huang SY. HDOCK: A web server for proteinprotein and protein-DNA/RNA docking based on a hybrid strategy. Nucleic Acids Res. 2017; 45:W365-W373

18. van Zundert GCP, Rodrigues J, Trellet M, Schmitz C, Kastritis PL, Karaca E, Melquiond ASJ, van Dijk M, de Vries SJ, Bonvin A. The HADDOCK2.2 web 
server: User-friendly integrative modeling of biomolecular complexes. J Mol Biol. 2016; 428:720-725

19. Schrödinger release 2017-2: Desmond molecular dynamics system, D. E. Shaw research, New York, NY, 2017. Maestro-desmond interoperability tools, schrödinger, New York, NY 2017;

20. Mattapally S, Singh M, Murthy KS, Asthana S, Banerjee SK. Computational modeling suggests impaired interactions between NKX2.5 and GATA4 in individuals carrying a novel pathogenic D16N NKX2.5 mutation. Oncotarget. 2018; 9:13713-13732

21. Xu Z, Taylor JA. SNPinfo: Integrating GWAS and candidate gene information into functional SNP selection for genetic association studies. Nucleic Acids Res. 2009; 37:W600-605

22. Bashey RI, Cox R, McCann J, Jimenez SA. Changes in collagen biosynthesis, types, and mechanics of aorta in hypertensive rats. J Lab Clin Med. 1989; 113:604-611

23. Intengan HD, Schiffrin EL. Vascular remodeling in hypertension: Roles of apoptosis, inflammation, and fibrosis. Hypertension. 2001; 38:581-587

24. Birkedal-Hansen $\mathrm{H}$. Proteolytic remodeling of extracellular matrix. Curr Opin Cell Biol. 1995; 7:728-735

25. Schiffrin EL, Touyz RM. From bedside to bench to bedside: Role of reninangiotensin-aldosterone system in remodeling of resistance arteries in hypertension. Am J Physiol Heart Circ Physiol. 2004; 287:H435-446

26. Pardo A, Selman M. Role of matrix metalloproteases in pulmonary fibrosis. In: Lagente V, Boichot E, eds. Matrix metalloproteinases in tissue remodelling and inflammation. Basel: Birkhäuser Basel; 2008:39-55.

27. Yang X, Liu Y, Yang Y, Li B. Update meta-analysis on MMP-7 -181a>G polymorphism and cancer risk: Evidence from 25 studies. Gene. 2013; 521:252258

28. Lu ZQ, Wang YM, Cao YY, Zhang QJ, Zhang XH, Li YH, Wang HS, Xie HL, Jiao $\mathrm{BH}$, Zhang $\mathrm{JH}$. Correlations of polymorphisms in matrix metalloproteinase-3 and 
-7 promoters to susceptibility to brain astrocytoma. Chinese journal of cancer. $2007 ; 26: 463-468$

29. Li Y, Jin X, Kang S, Wang Y, Du H, Zhang J, Guo W, Wang N, Fang S. Polymorphisms in the promoter regions of the matrix metalloproteinases-1, $-3,-7$, and -9 and the risk of epithelial ovarian cancer in China. Gynecol Oncol. 2006; 101:92-96

30. Singh $\mathrm{H}$, Jain $\mathrm{M}$, Mittal B. MMP-7 (-181a $>\mathrm{G})$ promoter polymorphisms and risk for cervical cancer. Gynecol Oncol. 2008; 110:71-75

31. Mishra A, Srivastava A, Mittal T, Garg N, Mittal B. Association of matrix metalloproteinases (MMP2, MMP7 and MMP9) genetic variants with left ventricular dysfunction in coronary artery disease patients. Clin Chim Acta. 2012; 413:1668-1674

32. Mishra A, Srivastava A, Mittal T, Garg N, Mittal B. Genetic predisposition to left ventricular dysfunction: A multigenic and multi-analytical approach. Gene. 2014; 546:309-317

33. Shaywitz AJ, Greenberg ME. CREB: A stimulus-induced transcription factor activated by a diverse array of extracellular signals. Annu Rev Biochem. 1999; 68:821-861

34. Mayr B, Montminy M. Transcriptional regulation by the phosphorylationdependent factor CREB. Nat Rev Mol Cell Biol. 2001; 2:599-609

35. Ichiki T. Role of cAMP response element binding protein in cardiovascular remodeling: Good, bad, or both? Arterioscler Thromb Vasc Biol. 2006; 26:449455

36. Fentzke RC, Korcarz CE, Lang RM, Lin H, Leiden JM. Dilated cardiomyopathy in transgenic mice expressing a dominant-negative CREB transcription factor in the heart. J Clin Invest. 1998; 101:2415-2426

37. Wellman GC, Cartin L, Eckman DM, Stevenson AS, Saundry CM, Lederer WJ, Nelson MT. Membrane depolarization, elevated $\mathrm{Ca}(2+)$ entry, and gene expression in cerebral arteries of hypertensive rats. Am J Physiol Heart Circ Physiol. 2001; 281 :H2559-2567 
38. Gerzanich V, Ivanova S, Simard JM. Early pathophysiological changes in cerebral vessels predisposing to stroke. Clin Hemorheol Microcirc. 2003; 29:291294

39. Funakoshi $\mathrm{Y}$, Ichiki T, Takeda K, Tokuno $\mathrm{T}$, lino N, Takeshita A. Critical role of cAMP-response element-binding protein for angiotensin II-induced hypertrophy of vascular smooth muscle cells. J Biol Chem. 2002; 277:18710-18717

40. Benbow $U$, Brinckerhoff CE. The AP-1 site and MMP gene regulation: What is all the fuss about? Matrix Biol. 1997; 15:519-526

41. Chakraborti S, Mandal M, Das S, Mandal A, Chakraborti T. Regulation of matrix metalloproteinases: An overview. Mol Cell Biochem. 2003; 253:269-285

42. Spinale FG. Myocardial matrix remodeling and the matrix metalloproteinases: Influence on cardiac form and function. Physiol Rev. 2007; 87:1285-1342

43. Gustavson MD, Crawford HC, Fingleton B, Matrisian LM. TCF binding sequence and position determines beta-catenin and Lef-1 responsiveness of MMP-7 promoters. Mol Carcinog. 2004; 41:125-139

44. Charmandari E, Tsigos C, Chrousos G. Endocrinology of the stress response. Annu Rev Physiol. 2005; 67:259-284

45. Shi M, Liu D, Duan H, Han C, Wei B, Qian L, Chen C, Guo L, Hu M, Yu M, Song L, Shen B, Guo N. Catecholamine up-regulates MMP-7 expression by activating AP-1 and STAT3 in gastric cancer. Mol Cancer. 2010; 9:269

46. Wang GL, Semenza GL. General involvement of hypoxia-inducible factor 1 in transcriptional response to hypoxia. Proc Natl Acad Sci U S A. 1993; 90:43044308

47. Hu CJ, Wang LY, Chodosh LA, Keith B, Simon MC. Differential roles of hypoxiainducible factor 1alpha (HIF-1alpha) and HIF-2alpha in hypoxic gene regulation. Mol Cell Biol. 2003; 23:9361-9374

48. Maxwell PH, Wiesener MS, Chang GW, Clifford SC, Vaux EC, Cockman ME, Wykoff CC, Pugh CW, Maher ER, Ratcliffe PJ. The tumour suppressor protein VHL targets hypoxia-inducible factors for oxygen-dependent proteolysis. Nature. 1999; 399:271-275 
49. Vaziri ND, Wang ZQ. Sustained systemic arterial hypertension induced by extended hypobaric hypoxia. Kidney Int. 1996; 49:1457-1463

50. Marina N, Ang R, Machhada A, Kasymov V, Karagiannis A, Hosford PS, Mosienko V, Teschemacher AG, Vihko P, Paton JF, Kasparov S, Gourine AV. Brainstem hypoxia contributes to the development of hypertension in the spontaneously hypertensive rat. Hypertension. 2015; 65:775-783

51. Calbet JA. Chronic hypoxia increases blood pressure and noradrenaline spillover in healthy humans. J Physiol. 2003; 551:379-386

52. Abe $\mathrm{H}$, Semba $\mathrm{H}$, Takeda $\mathrm{N}$. The roles of hypoxia signaling in the pathogenesis of cardiovascular diseases. J Atheroscler Thromb. 2017; 24:884-894

53. Goren I, Tavor E, Goldblum A, Honigman A. Two cysteine residues in the DNAbinding domain of CREB control binding to CRE and CREB-mediated gene expression. J Mol Biol. 2001; 313:695-709

54. Kvietikova I, Wenger $\mathrm{RH}$, Marti HH, Gassmann M. The transcription factors ATF1 and CREB-1 bind constitutively to the hypoxia-inducible factor-1 (HIF-1) DNA recognition site. Nucleic Acids Res. 1995; 23:4542-4550

55. Leonard MO, Howell K, Madden SF, Costello CM, Higgins DG, Taylor CT, McLoughlin P. Hypoxia selectively activates the CREB family of transcription factors in the in vivo lung. Am J Respir Crit Care Med. 2008; 178:977-983

56. Beitner-Johnson D, Millhorn DE. Hypoxia induces phosphorylation of the cyclic AMP response element-binding protein by a novel signaling mechanism. $J$ Biol Chem. 1998; 273:19834-19839

57. Williams SD, Ford DA. Calcium-independent phospholipase A(2) mediates CREB phosphorylation and c-fos expression during ischemia. Am J Physiol Heart Circ Physiol. 2001; 281:H168-176

58. Burke B, Giannoudis A, Corke KP, Gill D, Wells M, Ziegler-Heitbrock L, Lewis CE. Hypoxia-induced gene expression in human macrophages: Implications for ischemic tissues and hypoxia-regulated gene therapy. Am J Pathol. 2003; 163:1233-1243 


\section{Novelty and Significance}

\section{What is new?}

- This is the first study to the best of our knowledge demonstrating the association of MMP7 A-181G (rs11568818) polymorphism with hypertension risk.

- This study provides evidence for the role of CREB in the enhanced transcriptional activity of $M M P 7-181 \mathrm{G}$ promoter translating to higher protein levels in cardiomyocytes.

\section{What is relevant?}

The study identifies a highly prevalent common tag SNP playing a crucial role in conferring hypertension risk in Indian populations which may be used for developing preventive strategies in individuals likely to be predisposed to hypertension and its complications.

\section{Summary}

We identified a common naturally-occurring polymorphism (A-181G) in the upstream regulatory region of $M M P 7$ gene showing strong association with increased hypertension risk in two geographically distinct Indian populations comprising of $\sim 2500$ hypertensive and normotensive subjects using a systematic case-control approach. The $-181 \mathrm{G}$ allele containing promoter displayed higher promoter activity than the $-181 \mathrm{~A}$ promoter in basal as well as under pathophysiological stimuli (hypoxia, catecholamine excess) due to preferential binding with CREB transcription factor which translated to increased MMP7 levels in vitro. The risk genotype $(-181 \mathrm{~A} / \mathrm{G})$ also had a correlative association with increased plasma MMP7 levels and blood pressure suggesting that this functional regulatory polymorphism may act as predictor of cardiovascular risk. 


\section{TABLES}

Table 1. Occurrence of MMP7-181A/G polymorphism in Indian populations

\begin{tabular}{|c|c|c|c|c|c|c|}
\hline \multirow{2}{*}{$\begin{array}{c}\text { MMP7 } \\
\text { promoter } \\
\text { genotype }\end{array}$} & \multicolumn{3}{|c|}{ Occurrence in Chennai population } & \multicolumn{3}{|c|}{ Occurrence in Chandigarh population } \\
\hline & $\begin{array}{c}\text { HTN } \\
(n=862)\end{array}$ & $\begin{array}{l}\text { Controls } \\
(n=655)\end{array}$ & $\begin{array}{c}\text { Total } \\
(\mathrm{n}=1517)\end{array}$ & $\begin{array}{c}\text { HTN } \\
(n=519)\end{array}$ & $\begin{array}{l}\text { Controls } \\
(n=458)\end{array}$ & $\begin{array}{c}\text { Total } \\
(\mathrm{n}=977)\end{array}$ \\
\hline$A / A$ & 215 & 207 & 422 & 164 & 180 & 344 \\
\hline $\mathrm{A} / \mathrm{G}$ & 473 & 283 & 756 & 266 & 194 & 460 \\
\hline $\mathrm{G} / \mathrm{G}$ & 174 & 165 & 339 & 89 & 84 & 173 \\
\hline $\begin{array}{l}\text { Minor Allele } \\
\text { Frequency }\end{array}$ & 0.476 & 0.467 & 0.473 & 0.428 & 0.395 & 0.412 \\
\hline $\begin{array}{c}\mathrm{H}-\mathrm{W} \\
\mathrm{p} \text { value }\end{array}$ & & 0.991 & & & 0.372 & \\
\hline
\end{tabular}

Abbreviations: HTN, Hypertensive; H-W, Hardy-Weinberg equilibrium. 
Table 2. Association of MMP7-181A/G polymorphism with hypertension risk in Indian populations*

\begin{tabular}{|c|c|c|c|c|c|c|c|c|}
\hline \multirow{3}{*}{$\begin{array}{c}M M P 7 \\
\text { promoter } \\
\text { genotype }\end{array}$} & \multicolumn{4}{|c|}{ Chennai population $(n=1517)$} & \multicolumn{4}{|c|}{ Chandigarh population $(n=977)$} \\
\hline & \multicolumn{2}{|c|}{$\begin{array}{l}\text { Logistic Regression } \\
\qquad \text { (unadjusted) }\end{array}$} & \multicolumn{2}{|c|}{$\begin{array}{c}\text { Logistic Regression } \\
\text { (Age, Sex and BMI adjusted) }\end{array}$} & \multicolumn{2}{|c|}{$\begin{array}{l}\text { Logistic Regression } \\
\qquad \text { (unadjusted) }\end{array}$} & \multicolumn{2}{|c|}{$\begin{array}{l}\text { Logistic Regression } \\
\text { (Age and Sex adjusted) }\end{array}$} \\
\hline & OR (95\% Cl) & $p$ value & OR $(95 \% \mathrm{Cl})$ & $p$ value & OR (95\% Cl) & $\mathrm{p}$ value & OR $(95 \% \mathrm{Cl})$ & $p$ value \\
\hline $\mathrm{A} / \mathrm{A}$ & 1(Ref) & - & 1 (Ref) & - & 1(Ref) & - & 1 (Ref) & - \\
\hline$A / G$ & $1.675(1.315-2.133)$ & $3 \times 10^{-5}$ & $1.641(1.276-2.109)$ & $1 \times 10^{-4}$ & $1.505(1.136-1.993)$ & 0.004 & $1.520(1.106-2.090)$ & 0.010 \\
\hline $\mathrm{G} / \mathrm{G}$ & $1.059(0.796-1.411)$ & 0.693 & $1.017(0.757-1.367)$ & 0.911 & $1.163(0.807-1.676)$ & 0.418 & $1.314(0.858-2.014)$ & 0.209 \\
\hline $\mathrm{A} / \mathrm{G}+\mathrm{G} / \mathrm{G}$ & $1.447(1.154-1.815)$ & 0.001 & $1.408(1.113-1.781)$ & 0.004 & $1.402(1.077-1.824)$ & 0.012 & $1.464(1.085-1.977)$ & 0.013 \\
\hline
\end{tabular}

Abbreviations: OR, odds ratio; $\mathrm{BMI}$, body mass index; $\mathrm{Cl}$, confidence interval 


\section{FIGURES}

A

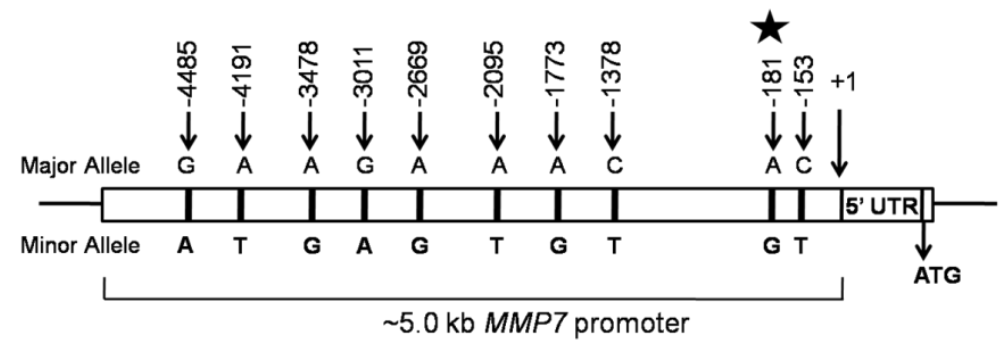

B
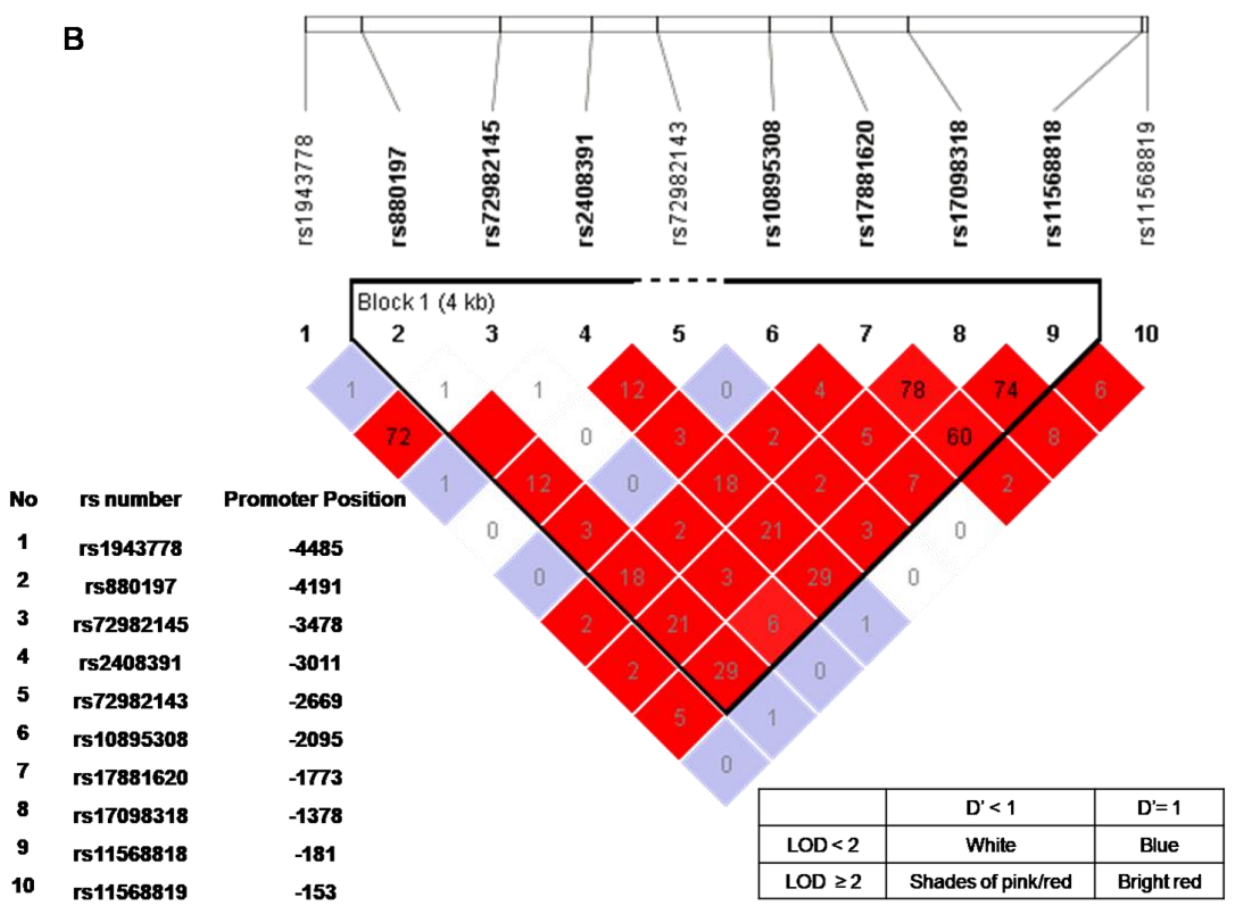

Figure 1. Common promoter polymorphisms in MMP7. (A) Schematic showing the common polymorphisms in the $5 \mathrm{~kb}$ promoter region of MMP7. MMP7 A-181G polymorphism was identified as a tag SNP. (B) MMP7 promoter polymorphisms and linkage disequilibrium (LD). LD plot of common polymorphisms in $5 \mathrm{~kb}$ upstream region of MMP7 promoter in the south Asian (SAS) super population of 1000 genomes project. Pairwise LD values were plotted between common SNPs in the MMP7 promoter using Haploview 4.2. D': Coefficient of LD; LOD: log of the likelihood odds ratio. 
A

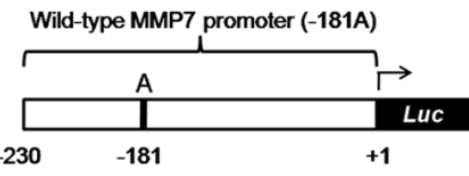

B

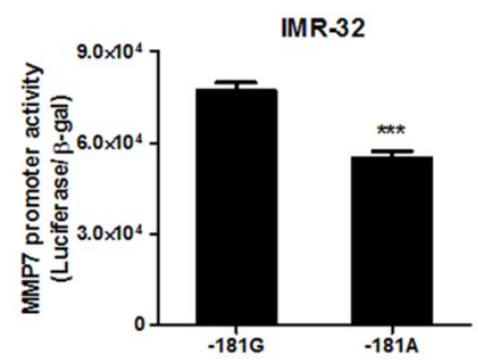

D

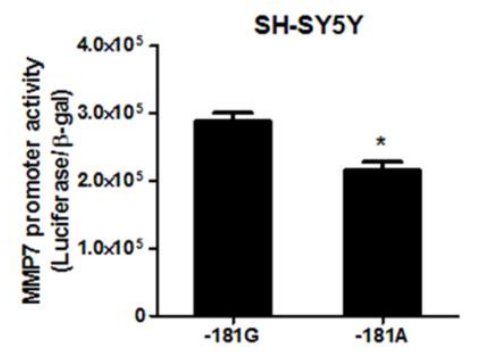

Variant MMP7 promoter (-181G)

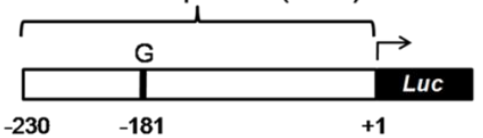

C

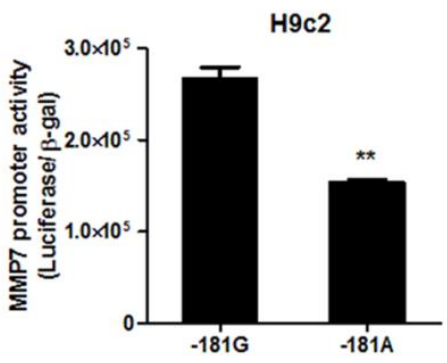

$\mathrm{E}$

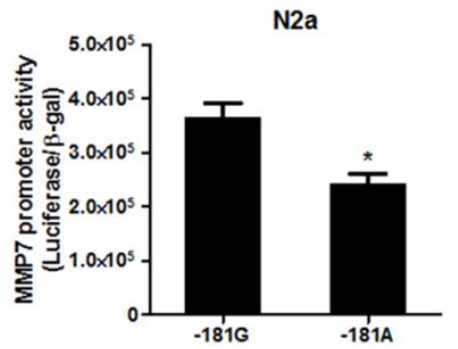

Figure 2. Differential promoter activities of $M M P 7$ promoter reporter constructs. (A) Schematic representation of the MMP7 promoter reporter constructs cloned into pGL3basic vector. (B - E) The MMP7 promoter region harboring the $-181 \mathrm{~A} / \mathrm{G}$ SNP from AA and GG individuals were cloned into pGL3-Basic plasmid. MMP7 -181G and -181A promoter reporter constructs were transfected into rat cardiomyoblast H9c2 (B) neuroblastoma cell lines IMR-32 (C), SH-SY5Y (D) and N2a (E) along with $\beta$ galactosidase expression plasmid. Results are expressed as mean \pm SEM of triplicate values of the ratio of luciferase/ $\beta$-gal activity. ${ }^{*} p<0.05$, ${ }^{* *} p<0.01$ and ${ }^{* * *} p<0.001$ when compared to $-181 \mathrm{G}$ construct. 

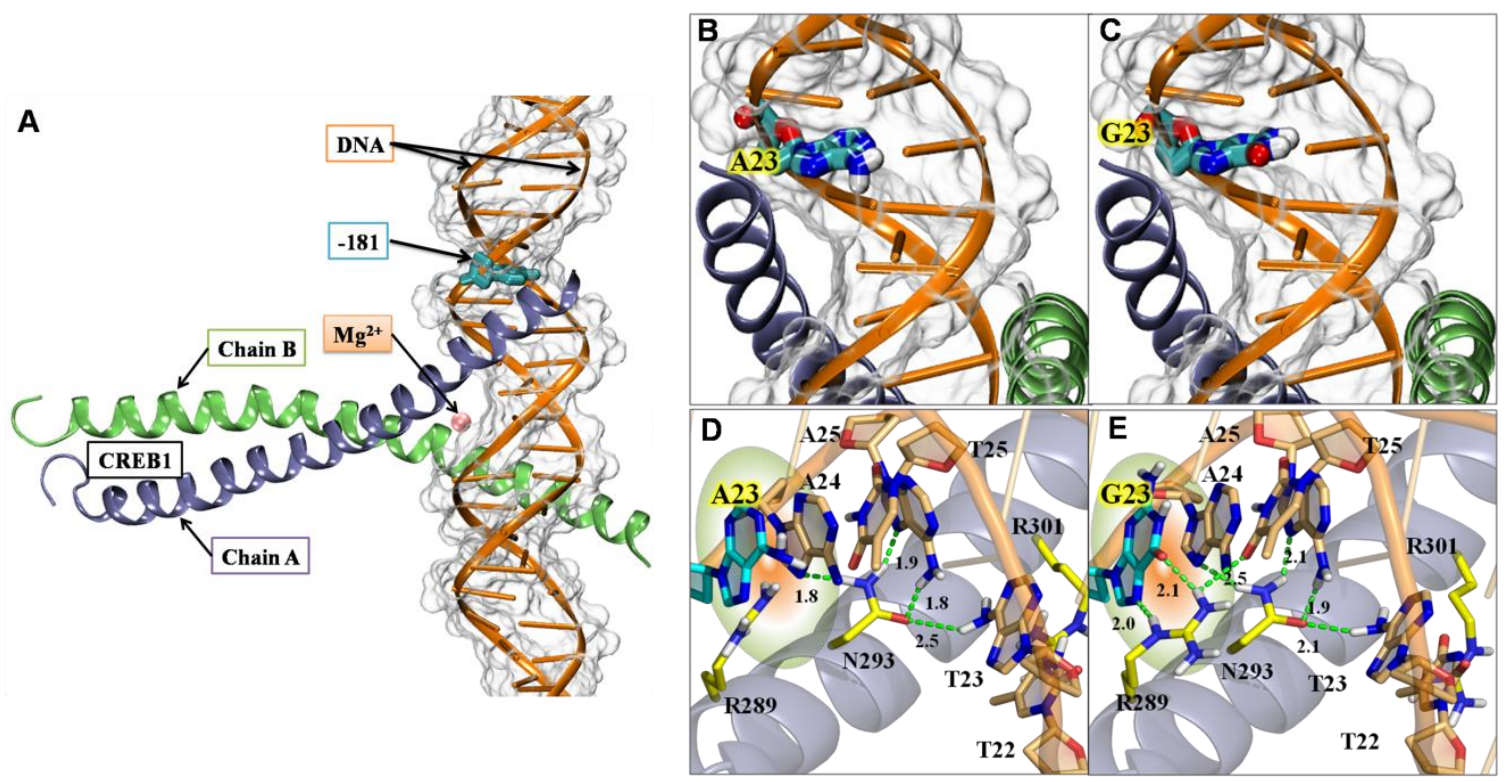

Figure 3. Schematic diagram of interactions of MMP7-promoter DNA with CREB1 transcription factor. (A) Representative energy minimized model of CREB1:wild-type MMP7 complex rendered in new cartoon representation. DNA is shown in orange color; CREB1-chain $A$ and CREB1-chain $B$ are shown in ice-blue and green colors, respectively. DNA is shown in transparent white surface view as well. Position of the $181 \mathrm{bp}$ nucleotide and $\mathrm{Mg}^{2+}$ has been indicated. (B) Positioning of the key nucleotide $181 \mathrm{~A}$ (labelled as A23) in wild-type promoter. (C) Positioning of key nucleotide -181G (labeled as G23) in the mutant promoter. (D) and (E) Comparison of wild-type and mutant MMP7-promoter DNA interactions with CREB1 in enlarged view. Amino acids are indicated by single letter codes and based on their positions in the CREB1-chain A. Amino acids and nucleotides are rendered in licorice and colored atom wise; C: light orange, $\mathrm{N}$ : blue, $\mathrm{O}$ : red, $\mathrm{H}$ : white. Hydrogen bonds are shown in green dotted lines. The mutant promoter (E) involves several additional hydrogen bonds as compared to the wild-type promoter (D) suggesting stronger interactions with CREB1. 


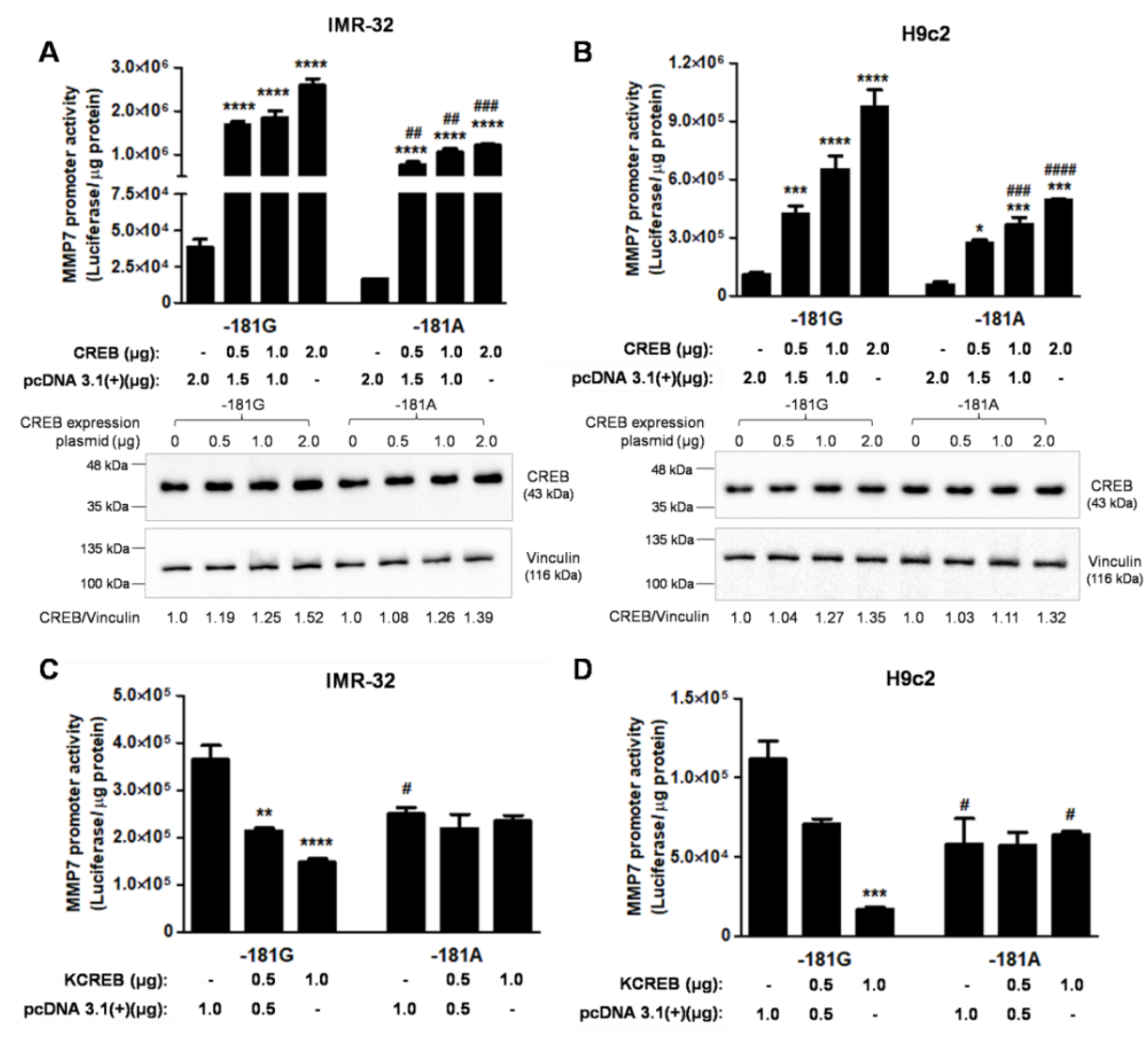

Figure 4. Role of CREB in augmentation of MMP7 promoter activity. IMR-32 (A) and H9c2 (B) cells were transiently transfected with MMP7 $-181 \mathrm{G}$ and $-181 \mathrm{~A}$ promoterreporter constructs and increasing doses of CREB expression plasmid. pcDNA 3.1(+) was used as balancing plasmid. Assays were performed 30 hours after transfection and results expressed as mean \pm SEM of triplicate values of the ratio of luciferase activity/ug protein. ${ }^{*} p<0.05,{ }^{* *} p<0.01$ and ${ }^{* * *} p<0.001$ when compared to basal activity of the

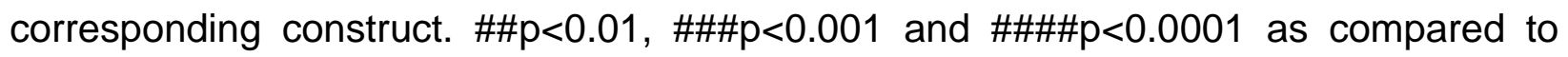
corresponding dose in case of the MMP7-181G construct. Over-expression of CREB was confirmed by western blotting. (C) and (D) Dominant negative KCREB diminishes the promoter activity of MMP7-181G promoter. IMR-32 (C) and H9c2 (D) cells were transiently transfected with MMP7 promoter-reporter constructs and increasing quantities of KCREB plasmid. pcDNA3.1(+) was used as balancing plasmid. Assays were performed 30 hours after transfection and results expressed as mean \pm SEM of triplicate values of the ratio of luciferase activity/ug protein. ${ }^{* *} p<0.01,{ }^{* * *} p<0.001$ and ${ }^{* * * *} p<0.0001$ when compared to basal activity of the corresponding construct. $\# p<0.05$ as compared to corresponding dose in case of the MMP7-181G construct. 

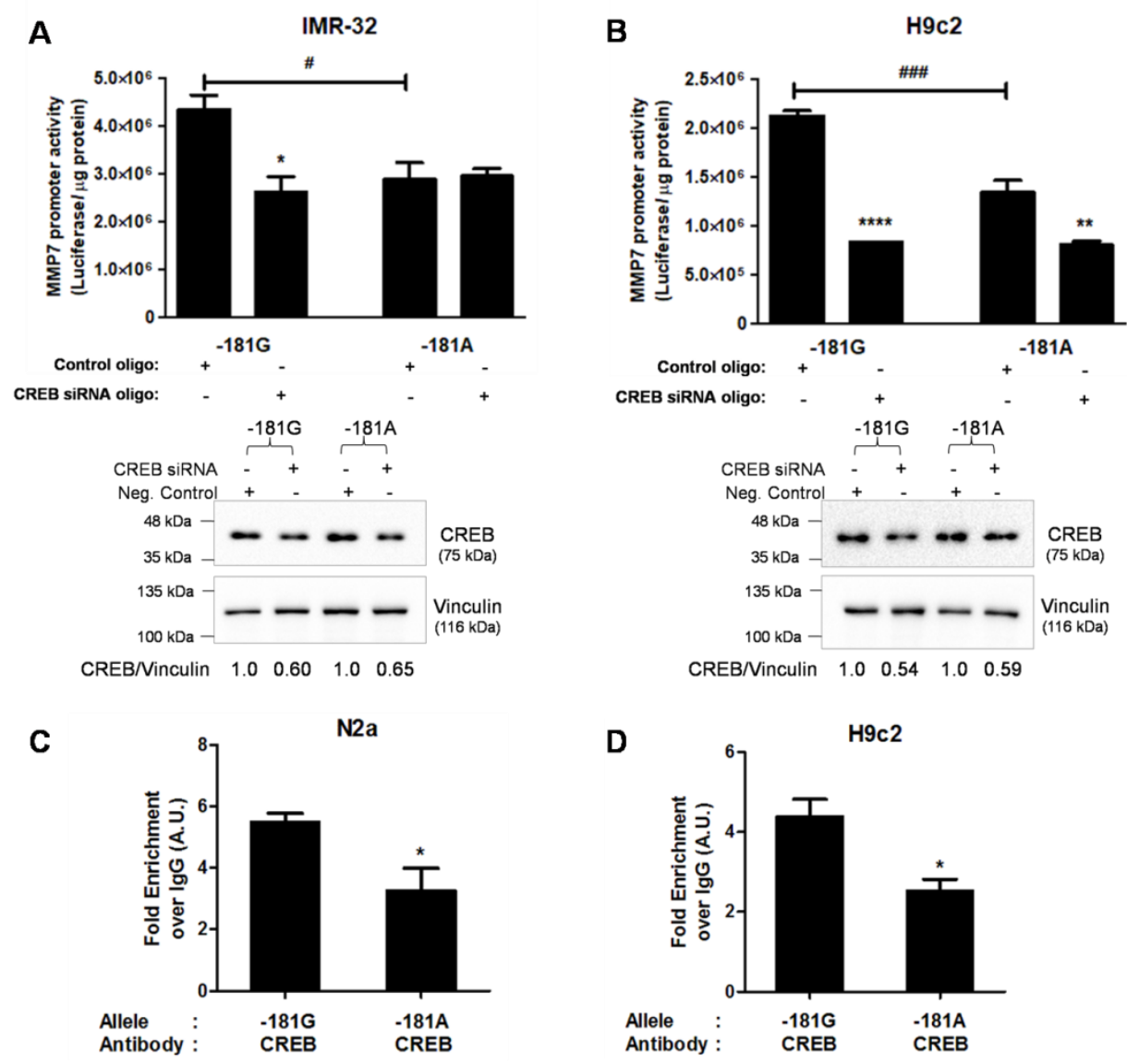

Figure 5. Enhanced interactions of CREB with $M M P 7-181 \mathrm{G}$ allele in vivo in the context of chromatin. (A) and (B) siRNA mediated knock-down of CREB decreases the MMP7$181 \mathrm{G}$ promoter activity. IMR-32 (A) and H9c2 (B) cells were transfected with MMP7 $181 \mathrm{G}$ or $-181 \mathrm{~A}$ constructs along with control siRNA oligo or CREB siRNA oligo. Assays were performed after 48 hours of transfection and results expressed as mean \pm SEM of triplicate values of the ratio of luciferase activity $/ \mu \mathrm{g}$ protein. ${ }^{*} p<0.05,{ }^{* *} p<0.01$ and ${ }^{* * * *} p<0.0001$ when compared to the basal activity of the corresponding construct. $\# p<0.05$ and \#\#\# $\mathrm{p}<0.001$ as compared to basal activity of MMP7-181G construct. Down-regulation of CREB was confirmed by western blotting. (C) and (D) Interaction of CREB with $-181 \mathrm{G} / \mathrm{A}$ alleles of MMP7 promoter. Binding of CREB to $-181 \mathrm{G}$ and $-181 \mathrm{~A}$ alleles of MMP7 promoter. Chromatin immunoprecipitation of N2a (C) and H9c2 (D) cells transfected with $-181 \mathrm{G}$ or $-181 \mathrm{~A}$ construct was carried out using antibody against CREB/control IgG. Immunoprecipitated chromatin was subjected to qPCR. Fold enrichment in case of CREB antibody over $\operatorname{lgG}$ control is shown. CREB displayed higher binding affinity towards $-181 \mathrm{G}$ promoter when compared to the $-181 \mathrm{~A}$ promoter. 

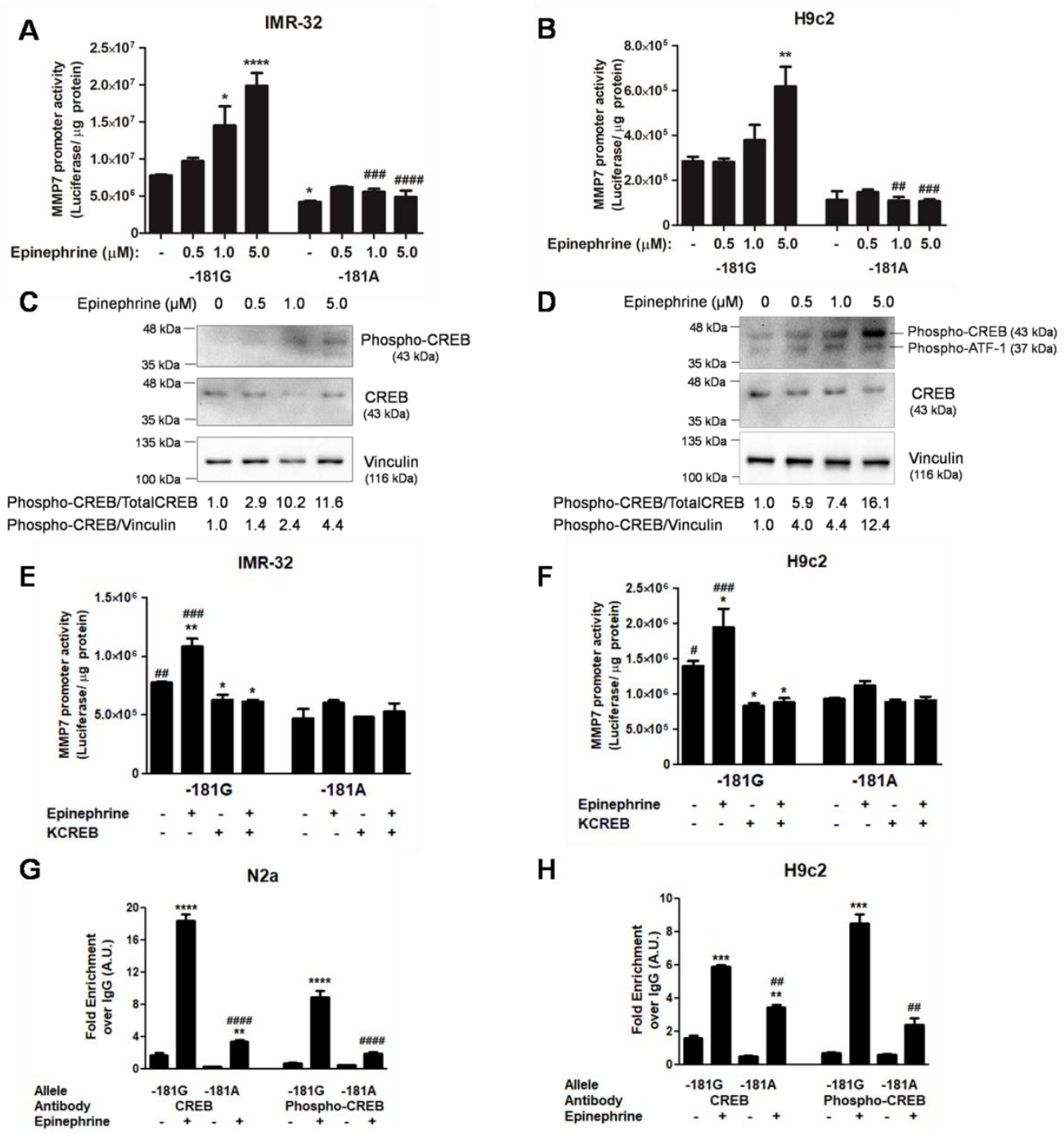

$\mathbf{F}$

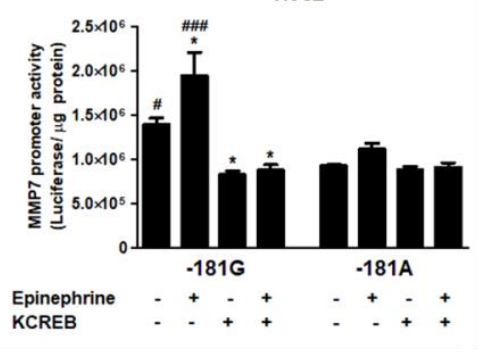

H

H9c2

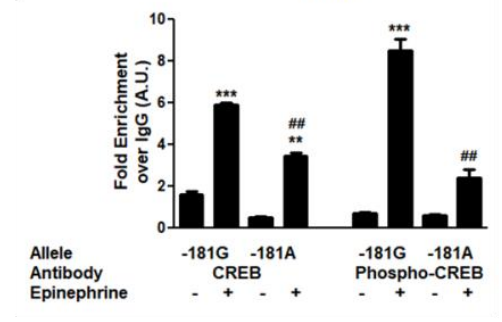

Figure 6. Epinephrine induced activation of $M M P 7-181 \mathrm{G}$ promoter via CREB. IMR-32 (A) and H9c2 (B) cells were transfected with MMP7 promoter $-181 \mathrm{G}$ and $-181 \mathrm{~A}$ constructs and treated with increasing doses of epinephrine for $6 \mathrm{hrs}$. Results are mean \pm SEM of triplicate values of luciferase activity/ $\mu$ g protein. ${ }^{*} p<0.05,{ }^{* *} p<0.01$ and ${ }^{* * * *} p<0.0001$ when compared to respective basal condition and $\# \# p<0.01, \# \# \# p<0.001$ and \#\#\#\#p<0.0001 when compared to the corresponding treatment in case of $-181 \mathrm{G}$ construct. (C) and (D) Effect of epinephrine treatment on CREB expression. IMR-32 (C) and H9c2 (D) cells were treated with increasing doses of epinephrine for $6 \mathrm{hrs}$ and western blot analysis of total proteins probing for phospho-CREB, CREB and Vinculin was carried out. $(E)$ and $(F)$ Role of CREB in allele specific activation of MMP7 promoter $-181 \mathrm{G}$ by epinephrine. MMP7 promoter $-181 \mathrm{G}$ and $-181 \mathrm{~A}$ constructs were transfected into IMR-32 (E) and H9c2 (F) cells with or without dominant negative KCREB plasmid followed by treatment with epinephrine $(5 \mu \mathrm{M})$ for 6 hrs. Results are 
expressed as mean \pm SEM of triplicate values of luciferase activity/ $\mu$ g protein. ${ }^{*} p<0.05$ and ${ }^{* *} p<0.01$ when compared to basal condition and $\# p<0.05, \# \# p<0.01$ and $\# \# \#$ < $<0.001$ when compared to the corresponding condition in $-181 \mathrm{~A}$ construct. $(\mathbf{G})$ and (H) ChIP assay of $\mathrm{N} 2 \mathrm{a}$ and $\mathrm{H} 9 \mathrm{c} 2$ cells transfected with MMP7 promoter $-181 \mathrm{G}$ or $-181 \mathrm{~A}$ construct, with or without epinephrine treatment was carried out using antibody against CREB, phospho-CREB and IgG. Immunoprecipitated chromatin was subjected to qPCR. Fold enrichment in signal obtained in case of CREB and phospho-CREB antibodies over IgG control is shown. ${ }^{* *} p<0.01,{ }^{* * *} p<0.001$ and ${ }^{* * * *} p<0.0001$ when compared to fold enrichment in respective untreated condition and $\# \# p<0.01$ and $\# \# \#$ P $<0.0001$ when compared to the corresponding treatment in case of $-181 \mathrm{G}$ construct. 
A

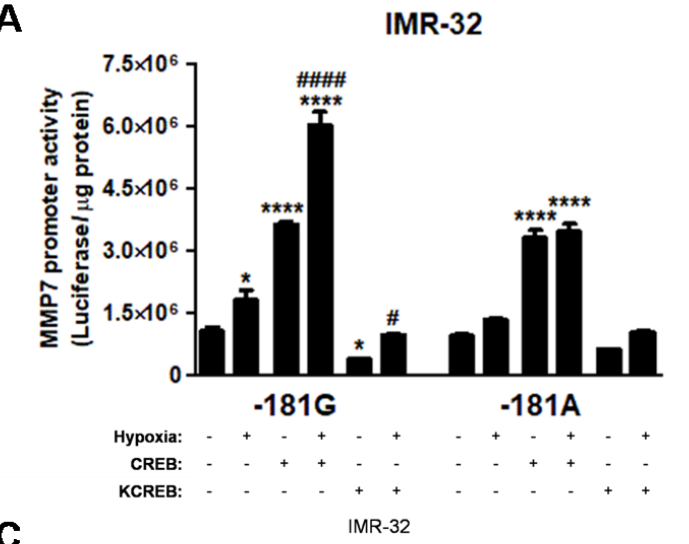

C

E

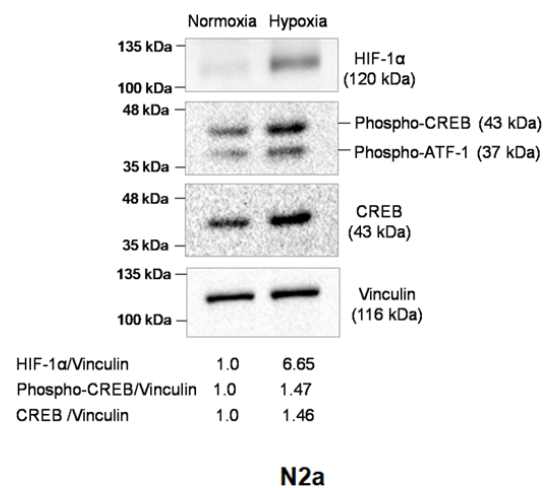

B

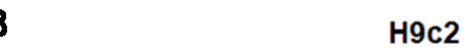

D

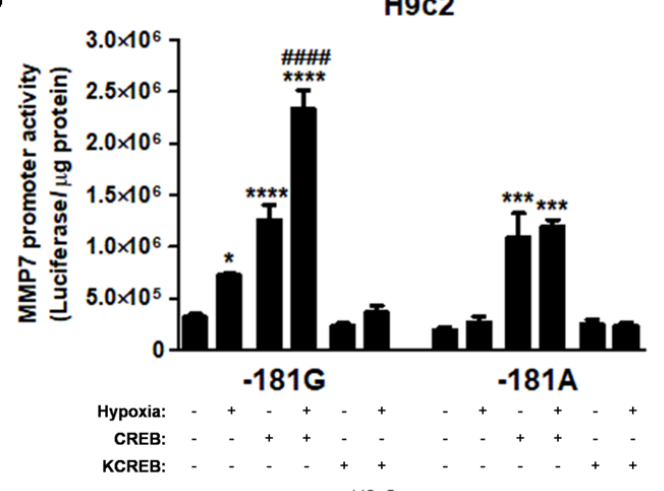

$\mathbf{F}$
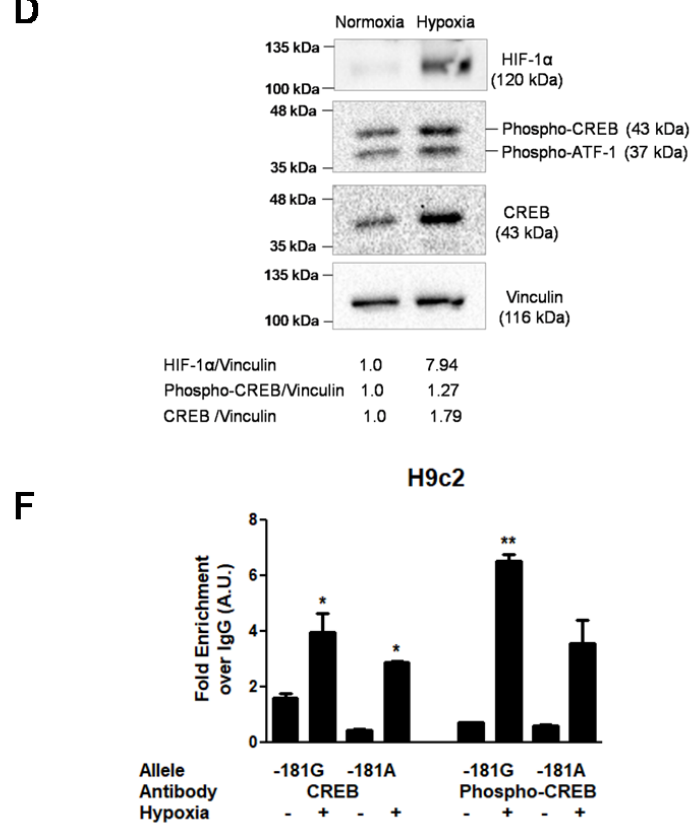

Figure 7. Effect of hypoxia on MMP7 promoter: crucial role for CREB. (A) and (B) Role of CREB in allele specific activation of MMP7 -181G promoter in response to hypoxia. MMP7 -181G and -181A promoter reporter constructs were transfected into IMR-32 (A) and H9c2 (B) cells with/without CREB and KCREB expression plasmid and subjected to 12 hrs of hypoxia. Results are expressed as mean \pm SEM of triplicate values of luciferase activity/ug protein, ${ }^{*} p<0.05,{ }^{* * *} p<0.001$ and ${ }^{* * * *} p<0.0001$ when compared to basal activity of the respective construct and $\# p<0.05$ and $\# \# \# \#<<0.0001$ when compared to the corresponding normoxic condition. (C) and (D) Effect of hypoxia on CREB expression. IMR-32 (C) and H9c2 (D) cells were subjected to hypoxia for 12 hrs and western blotting of the total proteins was carried out probing for HIF1- $\alpha$, CREB, phospho-CREB and vinculin. (E) and (F) Effect of hypoxia on the binding of CREB to MMP7 $-181 \mathrm{G}$ and $-181 \mathrm{~A}$ alleles. Chromatin immunoprecipitation of $\mathrm{N} 2 \mathrm{a}$ and $\mathrm{H} 9 \mathrm{c} 2$ cells transfected with MMP7 $-181 \mathrm{G}$ or $-181 \mathrm{~A}$ promoter construct, with/without exposure to hypoxia was carried out using antibody against CREB, phospho-CREB and pre-immune 
IgG. Immunoprecipitated chromatin was subjected to QPCR and fold enrichment in signal obtained in case of $\mathrm{CREB} /$ phospho-CREB antibody over pre-immune lgG is shown. ${ }^{*} p<0.05{ }^{* *} p<0.01,{ }^{* * *} p<0.001$ and ${ }^{* * *} p<0.0001$ when compared to fold enrichment in respective untreated condition and \#\#\#\#p<0.0001 as compared to the corresponding treatment in case of MMP7 $-181 \mathrm{G}$ promoter construct. 
A

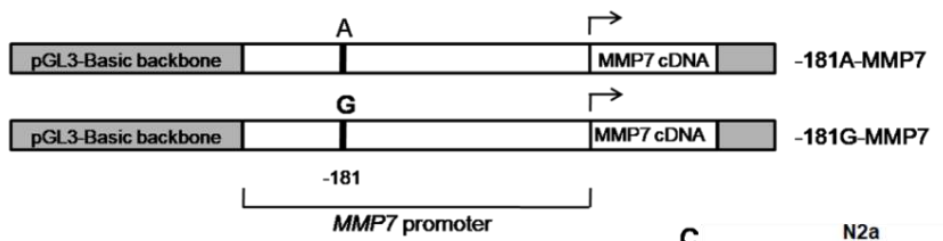

B

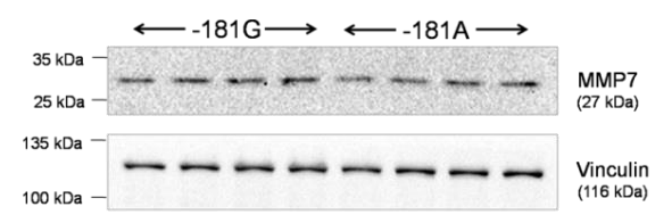

MMP7/Vinculin $\begin{array}{llllllll}1.71 & 1.59 & 1.61 & 1.86 & 1.02 & 1.05 & 1.14 & 1.02\end{array}$

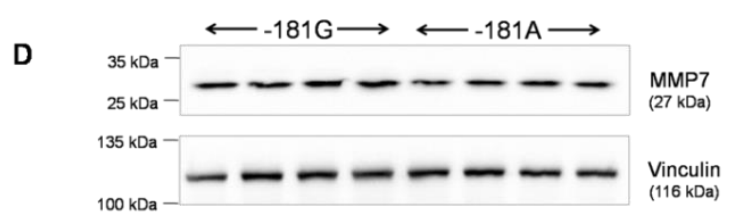

C

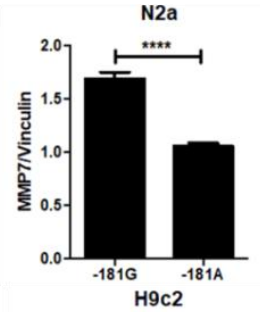

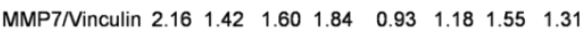

$E$
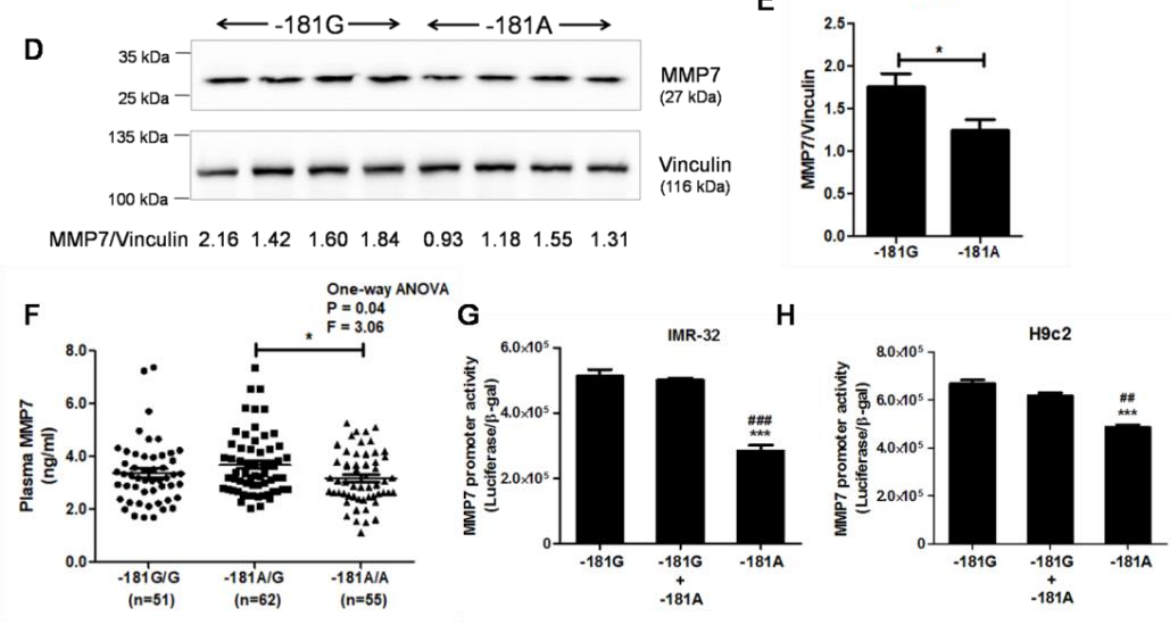

Figure 8. MMP7 -181G promoter confers higher expression of MMP7 in vitro. (A) Schematic representation of the MMP7 promoter-cDNA constructs. The MMP7 cDNA was subcloned in frame into the $-181 \mathrm{G}$ and $-181 \mathrm{~A}$ promoter reporter constructs replacing the luciferase cDNA. (B) and (C) In vitro expression of MMP7 under the influence of MMP7 -181G and -181 A promoters in N2a cells. N2a cells were transfected with -181G-MMP7 and -181A-MMP7 constructs and western blotting (B) of total proteins was carried out to probe for MMP7 and vinculin. Fold difference (MMP7/vinculin) is indicated (C). (D) and (E) In vitro expression of MMP7 under the influence of MMP7 $-181 \mathrm{G}$ and $-181 \mathrm{~A}$ promoters in $\mathrm{H} 9 \mathrm{c} 2$ cells. H9c2 cells were transfected with -181G-MMP7 and -181A-MMP7 constructs and western blotting (D) of total proteins was carried out to probe for MMP7 and vinculin. Fold difference (MMP7/vinculin) is indicated (E). (F) Plasma MMP7 levels in $-181 \mathrm{~A} / \mathrm{A},-181 \mathrm{~A} / \mathrm{G}$ and $181 \mathrm{G} / \mathrm{G}$ individuals as measured by ELISA. MMP7 $-181 \mathrm{~A} / \mathrm{G}$ individuals presented with significantly higher MMP7 levels. (G) and (H) MMP7-181A/G promoter activities in diploid combinations in cella. IMR-32 $(\mathrm{G})$ and $\mathrm{H} 9 \mathrm{c} 2 \mathrm{(H)}$ cells were transfected with $1 \mu \mathrm{gg}$ each of MMP7 $-181 \mathrm{G}$ or $-181 \mathrm{~A}$ or $500 \mathrm{ng}$ each of $-181 \mathrm{G}$ and $-181 \mathrm{~A}$ constructs. Results are mean \pm SEM of triplicate values of luciferase/ $\beta$-gal activity. ${ }^{* *} p<0.001$ as compared

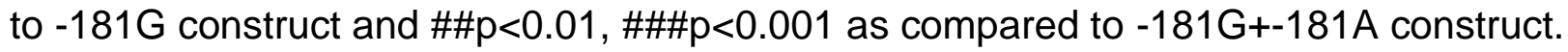


A

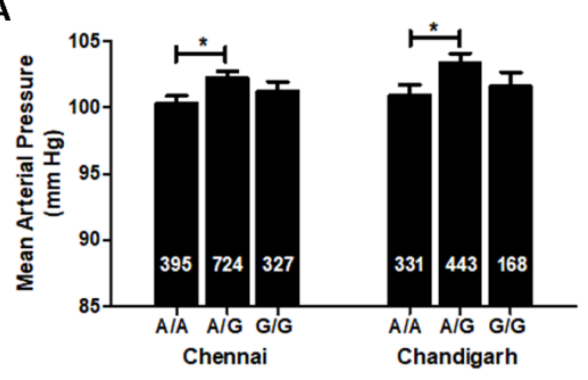

C

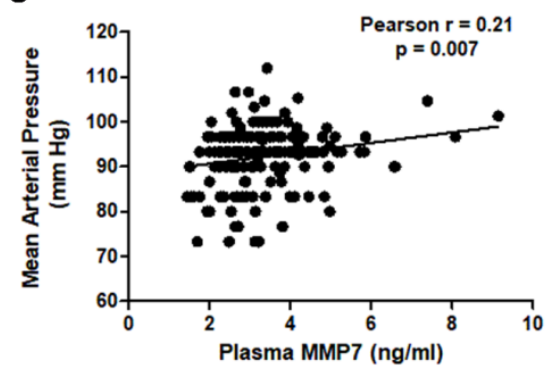

B

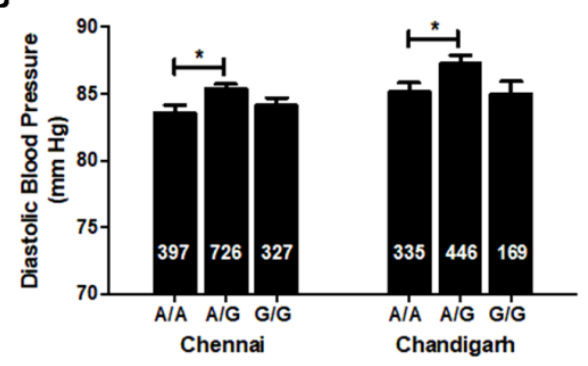

D

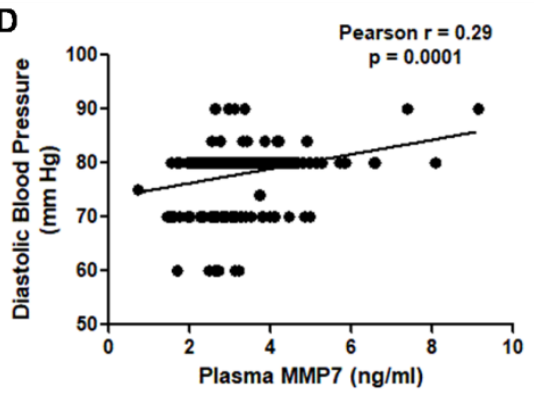

Figure 9. Allele-specific association of MMP7-181 promoter genotypes with blood pressure in Indian populations. (A) and (B) MMP7-181A/G individuals display higher mean arterial pressure (A) and diastolic blood pressure (B) in Chennai and Chandigarh populations. (C) and (D) Correlation of diastolic blood pressure (C) and mean arterial pressure (D) with MMP7 levels. MMP7 levels showed significant positive correlation with systolic, diastolic blood pressure and mean arterial pressure. Pearson $r$ and $p$ values for the correlations are indicated. 


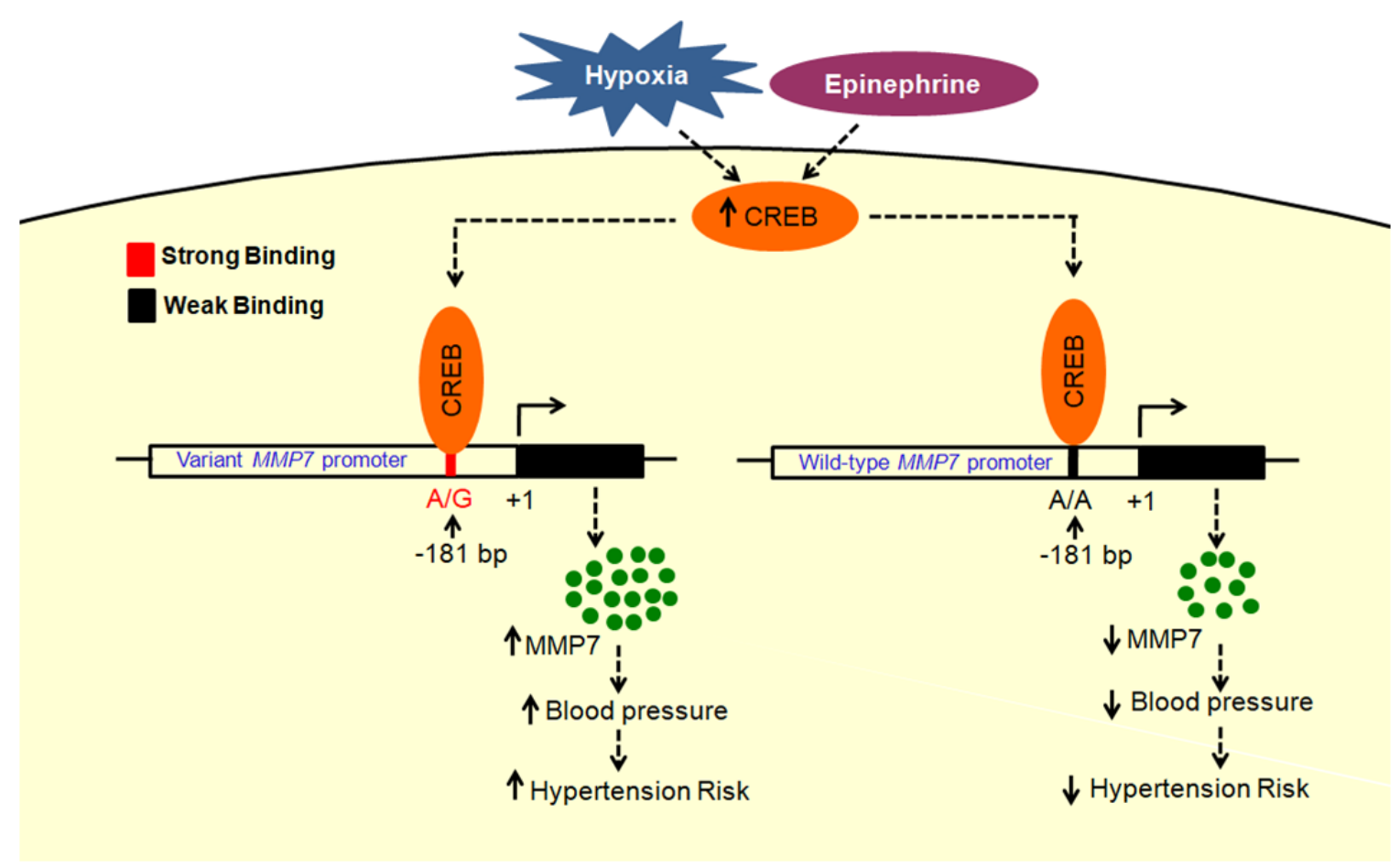

Figure 10. A schematic illustration of the plausible role and mechanistic basis behind the role of MMP7 -181 A/G promoter variant in contributing to hypertension risk. The MMP7 -181A/G genotype was found to be associated with increased hypertension risk and elevated blood pressure in the study population with the MMP7-181G promoter showing higher promoter activity consistently among different cell lines due to transcriptional activation by CREB. Pathophysiological conditions (viz. catecholamine excess and hypoxia) further increase the promoter activity of MMP7-181G construct in an allele-specific manner by activating CREB and inducing its phosphorylation. The higher promoter activity of MMP7-181G construct corroborates with the elevated levels of plasma MMP7 observed in $-181 \mathrm{~A} / \mathrm{G}$ individuals which, in turn, positively correlates with blood pressure suggesting that individuals heterozygous for the $M M P 7-181 \mathrm{~A} / \mathrm{G}$ genotype may be at a higher risk of hypertension and its complications. 


\title{
Online Data Supplement
}

\section{A common tag nucleotide variant in MMP7 promoter increases risk for hypertension via enhanced interactions with CREB transcription factor}

\author{
by \\ Lakshmi Subramanian ${ }^{1}$, Sakthisree Maghajothi ${ }^{1}$, Mrityunjay Singh ${ }^{2}$, Kousik Kesh ${ }^{3}$, \\ Kalyani Ananthamohan ${ }^{1}$, Saurabh Sharma ${ }^{4}$, Madhu Khullar ${ }^{4}$, Suma Victor ${ }^{5}$, Snehasikta \\ Swarnakar ${ }^{3}$, Shailendra Asthana ${ }^{2}$, Ajit S. Mullasari ${ }^{5}$, Nitish R. Mahapatra ${ }^{1}$
}

\section{from the}

${ }^{1}$ Department of Biotechnology, Bhupat and Jyoti Mehta School of Biosciences, Indian Institute of Technology Madras, Chennai - 600036, India

${ }^{2}$ Drug Discovery Research Center, Translational Health Science and Technology Institute, NCR Biotech Science Cluster, Faridabad, Haryana - 121001, India

${ }^{3}$ Drug Development Diagnostic and Biotechnology Division, Indian Institute of Chemical Biology, Kolkata - 700032, India

${ }^{4}$ Department of Experimental Medicine and Biotechnology, Postgraduate Institute of Medical Education and Research, Chandigarh - 160012, India

${ }^{5}$ Institute of Cardiovascular Diseases, Madras Medical Mission, Chennai - 600037, India

Running title: $M M P 7$ promoter polymorphism and hypertension risk

Address for correspondence: Dr. Nitish R. Mahapatra, Department of Biotechnology, Bhupat and Jyoti Mehta School of Biosciences, Indian Institute of Technology Madras, Chennai - 600036, India. Tel: 91-44-2257-4128; E-mail: nmahapatra@iitm.ac.in 


\section{DETAILED METHODS}

\section{Human Subjects}

A total of 2494 unrelated volunteers were enrolled in this case-control study. The primary study population comprised of 862 hypertensive cases (SBP $\geq 140 \mathrm{~mm} \mathrm{Hg}$ or DBP $\geq 90 \mathrm{~mm} \mathrm{Hg}$ or a history of hypertension or antihypertensive treatment but no history of cancer or kidney disease) and 655 normotensive controls (with no history of hypertension, diabetes, cancer or kidney disease) from an urban Chennai population recruited at the Madras Medical Mission (MMM), Chennai. The replication study population consisted of 519 hypertensive and 458 normotensive volunteers from Chandigarh recruited at the Postgraduate Institute of Medical Education and Research (PGIMER), Chandigarh. Informed written consent was obtained from each subject and the study was approved by the institutional ethics committee of Indian Institute of Technology Madras. Demographic details (age, gender), physical measurements (height, weight, and body mass index [BMI]), physiological parameters (systolic blood pressure [SBP], diastolic blood pressure [DBP], mean arterial pressure [MAP], heart rate $[H R]$ ), biochemical parameters (hemoglobin, sodium, potassium, urea, creatinine, blood sugar, total cholesterol [TC], triglycerides [TGL], low density lipoproteins [LDL] and high density lipoproteins [HDL]) wherever collected from the subjects have been listed in Supplementary Tables 1 and 2). The medical history of the volunteers (current medication, family history of cardiovascular or renal disease states) was recorded the time of recruitment. Blood samples were collected in EDTA coated tubes by venipuncture for genomic DNA isolation and plasma samples were collected, aliquoted and stored at $-80^{\circ} \mathrm{C}$ for assaying various biochemical parameters. The average age of the cases and controls was $\sim 44$ and $\sim 42$ years in the Chennai study population and $\sim 49$ and $\sim 59$ years in the Chandigarh population respectively. The BMI, blood pressure, sodium levels and triglyceride levels were significantly higher in hypertensive cases than normotensive controls across both the populations.

\section{Genotyping of MMP7-181A/G polymorphism in Indian populations}

Genomic DNA was isolated from heparin/EDTA-anticoagulated blood samples using Flexigene DNA kit (Qiagen, USA) according to manufacturer's protocol. The region of 
MMP7 spanning -302 bp to -153bp (NCBI accession number: NM_002423.4; numbered upstream (-) with respect to the cap site) was amplified using the primers: forward, hMMP7pro-181FP (5'-TGGTACCATAATGTCCTGAATG-3') and reverse, hMMP7pro181RP (5'-TCGTTATTGGCAGGAAGCACACAAGAATT-3'). The polymerase chain reaction was performed in Eppendorf Mastercycler. The 150 bp region was amplified in a $10 \mu \mathrm{l}$ reaction mixture containing $1 \mathrm{mM}$ concentrations of forward and reverse primers, $1.5 \mathrm{mM} \mathrm{MgCl} 2,0.4 \mathrm{mM}$ of each dNTP (New England Biolabs, USA), $20 \mathrm{ng}$ of the template and 0.4 units of the Phusion ${ }^{\mathrm{TM}}$ high fidelity DNA polymerase (New England Biolabs, USA). The reaction conditions included an initial denaturation of 2 min at $98^{\circ} \mathrm{C}$ followed by 35 cycles of denaturation at $98^{\circ} \mathrm{C}$ for $30 \mathrm{~s}$, annealing at $56.5^{\circ} \mathrm{C}$ for $30 \mathrm{~s}$ and extension at $72^{\circ} \mathrm{C}$ for $30 \mathrm{~s}$ with a final extension step at $72^{\circ} \mathrm{C}$ for $10 \mathrm{~min}$. The PCR products were digested with EcoRI enzyme and the digested products were electrophoresed in a $2.5 \%$ agarose gel. The genotypes were inferred by observed the digested band pattern. The presence of variant ( $G$ allele) creates a restriction site for EcoRI which digests the $150 \mathrm{bp}$ PCR product to yield two fragments of size $120 \mathrm{bp}$ and $30 \mathrm{bp}$, while the wild-type (A allele) remains undigested. Heterozygous (A/G) genotype results in three bands of sizes $150 \mathrm{bp}, 120 \mathrm{bp}$ and $30 \mathrm{bp}$ respectively. About $10 \%$ of the PCR products were also purified and sequenced by Sanger sequencing to confirm the inferred genotypes.

\section{Estimation of biochemical parameters}

Biochemical parameters such as random blood sugar, total cholesterol, triglycerides, LDL, HDL, urea, creatinine, hemoglobin, sodium and potassium levels were measured in the plasma using standard biochemical assays. The blood pressure readings were measured by experienced nursing staff as an average of triplicate values recorded in the sitting position using a brachial oscillometric cuff. Plasma MMP7 levels were measured by a commercially available immunoassay kit (R\&D systems, USA) following the manufacturer's protocol.

\section{Cloning and mutagenesis}

The 250 bp promoter region of MMP7 was amplified by PCR using genomic DNA of known homozygotes for MMP7-181A/G polymorphism and the following primers: 
hMMP7-230-FP: 5'-CGGGGTACCTGGAGTCAATTTATGCAGCAGACAG-3' and hMMP7+22-RP: 5'-CCGCTCGAGTTGGACCTATGGTTGATTTGGTG-3' (restriction sites of Kpnl and Xhol inserted at the 5 ' end of forward and reverse primers respectively are indicated in boldface). The purified promoter fragments were inserted between Kpnl and Xhol sites in the promoterless luciferase reporter vector pGL3-Basic which has the firely luciferase as the reporter gene (New England Biolabs, USA). The generated promoter-reporter constructs consisted of the -230- to +22-bp region of MMP7. Accurate cloning of the inserts with the variation was confirmed by DNA sequencing using the primers: RVprimer3 5'-CTAGCAAAATAGGCTGTCCC-3' and GLprimer2 5'CTTTATGTTTTTGGCGTCTTCCA-3'.

To generate the MMP7 promoter haplotype-cDNA constructs, MMP7 cDNA was subcloned from pcDNA3-GFP-MMP7, a gift from Steven Johnson (Addgene plasmid \# 11989) into the MMP7-181G and MMP7-181A promoter-reporter constructs replacing the luciferase cDNA. The MMP7-181G and MMP7-181A constructs were digested with Ncol followed by mungbean nuclease treatment to blunt the sticky end and then digested with Xbal to release the $1.6 \mathrm{~kb}$ luciferase cDNA. The pcDNA3-GFP-MMP7 plasmid was digested with EcoRI followed by mungbean nuclease treatment to blunt the sticky end and then digested with Xbal to release the 804 bp MMP7 cDNA. The 804 bp MMP7 cDNA was then ligated into the $3.1 \mathrm{~kb}$ backbone of MMP7-181G and MMP7$181 \mathrm{~A}$ constructs. The clones were confirmed by sequencing using primers hMMP7-230FP: 5'-CGGGGTACCTGGAGTCAATTTATGCAGCAGACAG-3' and pGL3pro RP: 5'CCCCCTGAACCTGAAACATA-3'.

All the plasmids were purified on columns using an endotoxin-free plasmid DNA purification kit (Hi Media, India) for transfection experiments.

\section{Cell culture, transfection and reporter assays}

Human neuroblastoma IMR-32, SH-SY5Y, rat cardiomyoblast $\mathrm{H} 9 \mathrm{c} 2$ and mouse neuroblastoma N2a cell lines were obtained from the National Center for Cell Sciences, Pune, India. Cell lines were maintained in Dulbecco's Modified Eagle Medium (DMEM) containing high glucose, L-glutamine and sodium pyruvate (HyClone, USA) supplemented with $10 \%(\mathrm{v} / \mathrm{v})$ fetal bovine serum (Gibco, USA), penicillin G (100 U/ml) 
and streptomycin sulfate $\left(100 \mathrm{mg} / \mathrm{ml}\right.$ ) (Gibco, USA) in humidified incubators at $37^{\circ} \mathrm{C}$ with $5 \% \mathrm{CO}_{2}$ (Thermo Scientific, USA). Cells were grown up to $70 \%$ confluence in 24 well plates and transfections were performed in triplicates with $1.0 \mu \mathrm{g} /$ well of promoterreporter plasmid and $300 \mathrm{ng} /$ well of a $\beta$-galactosidase expression plasmid (as internal control). Cells were transfected using Targefect F2 transfection reagent (Targeting Systems, USA) following manufacturer's protocol. The culture media was changed to fresh complete medium after $6 \mathrm{hrs}$. After $30 \mathrm{hrs}$ of transfection, cells were lysed and luciferase and $\beta$-galactosidase assays were performed with the cell lysates as described previously. ${ }^{1}$ Promoter activities were expressed as luciferase/ $\beta$-galactosidase readings.

Co-transfection experiments were carried out in IMR-32 and H9c2 cells with different doses of CREB expression plasmid (a kind gift from Dr. David Ginty, Howard Hughes Medical Institute, USA) ${ }^{2}$ along with MMP7-181G and MMP7-181A promoterreporter constructs. pcDNA3.1(+) was used as balancing plasmid. Similarly cotransfection experiments with different doses of dominant negative KCREB expression plasmid (a kind gift from Dr. Richard H. Goodman, Vollum Institute, Oregon Health Sciences University, USA) ${ }^{2}$ along with MMP7-181G and MMP7-181A promoter-reporter constructs were carried out in IMR-32 and H9c2 cells. Human CREB dicer substrate siRNA oligos (sense, 5'-CCACCAAUCUGCUUCCUGUUUCUTT-3' and antisense, 5'AAAGAAACAGGAAGCAGAUUGGUGGUU-3') and negative control siRNA oligos (Integrated DNA Technologies, Belgium) were co-transfected in 12-well plates with MMP7-181G and MMP7-181A promoter-reporter plasmids using Targefect F2 transfection reagents (Targeting Systems, USA) into IMR-32 cells. Similarly, CREB dicer substrate siRNA oligos (sense, 5'-AAAGAAACAGGAAGCAGAUUGGUGGUU-3' and antisense, 5'-AAGGAAUUUCCCAAGUUGCUGAUACCC-3') were used for siRNA co-transfection experiments in $\mathrm{H} 9 \mathrm{c} 2$ cells. In all co-transfection experiments, cell lysates were assayed for luciferase activity $36 \mathrm{hrs}$ post-transfection. Total protein per individual well was also estimated in the corresponding cell lysates by using Bradford reagent (Bio-Rad, USA) and reporter activities were expressed as luciferase activity/ug of protein. 
In another set of experiments, IMR-32 and H9c2 cells transfected with the MMP7-181G and MMP7-181A promoter-reporter constructs were treated with different concentrations of epinephrine $(0.5,1.0$ and $5.0 \mu \mathrm{M})$ in serum free media after $24 \mathrm{hrs}$ of transfection. In some experiments, KCREB expression plasmid (1.0 $\mu \mathrm{g} / \mathrm{well})$ was cotransfected in IMR-32 and H9c2 cells prior to treatment with epinephrine (5.0 $\mu \mathrm{M})$. Cell lysates were assayed for luciferase activity after $6 \mathrm{hrs}$ of treatment with epinephrine. The promoter activities were normalized with total protein.

For hypoxia experiments, MMP7-181G and MMP7-181A promoter-reporter constructs were transfected into IMR-32 and H9c2 cells alone or co-transfected with CREB or KCREB expression plasmid and after 24 hrs of transfection, culture media was changed to serum free media and the cells to be maintained under hypoxic condition were transferred to a desiccator cabinet (Bel-Art, USA) which was flushed with argon gas for $3 \mathrm{~min}$ and sealed. The cells under this hypoxic condition were placed in an incubator at $37^{\circ} \mathrm{C}$. In parallel, for normoxic condition, 24 hrs after transfection, culture media was replaced with serum free media and the cells were transferred to a regular $\mathrm{CO}_{2}$ incubator. Luciferase and Bradford assays were performed after $12 \mathrm{hrs}$ of hypoxia.

In certain experiments, MMP7 promoter-reporter plasmids were transfected in diploid combinations to imitate the homozygous and heterozygous conditions in cella. IMR32 and H9c2 cells were transfected with $1 \mu \mathrm{g}$ each of MMP7-181G or MMP7-181A or $500 \mathrm{ng}$ each of MMP7-181G and MMP7-181A constructs along with $\beta$-galactosidase expression plasmid. Luciferase and $\beta$-galactosidase assays were performed after $24 \mathrm{hrs}$ of transfection. Promoter activities were expressed as luciferase/ $\beta$-galactosidase readings.

In another set of experiments, -181G-MMP7cDNA and -181A-MMP7cDNA constructs were transfected into $\mathrm{N} 2 \mathrm{a}$ and $\mathrm{H} 9 \mathrm{c} 2$ cells and the MMP7 levels in the protein lysates were estimated after 48 hrs of transfection by western blotting to assess the differences in transcription of MMP7 under the influence of MMP7-181A/G SNP.

\section{Western blotting}

Over-expression or down-regulation of CREB, phospho-CREB and HIF-1a after transfection experiments/epinephrine treatment/hypoxia was determined by western blotting. The cells were lysed in a buffer containing $50 \mathrm{mM}$ Tris- $\mathrm{HCl}(\mathrm{pH} 7.4), 150 \mathrm{mM}$ 
$\mathrm{NaCl}, 1 \%$ Triton X-100, 1\% sodiumdeoxycholate, 1 mM EDTA, $0.1 \%$ SDS, $1 \mathrm{mM}$ PMSF, protease inhibitor mixture (Sigma) and phosphatase inhibitors (1 $\mathrm{mM}$ sodium orthovanadate, $1 \mathrm{mM}$ sodium pyrophosphate and $50 \mathrm{mM}$ sodium fluoride) followed by sonication. The protein concentrations were estimated by Bradford assay (Bio-Rad, USA). Also, MMP7 promoter-cDNA constructs were transfected into N2a and H9c2 cells and the total protein was isolated after $48 \mathrm{hrs}$ of transfection and subjected to western blotting. Equal amount of protein samples $(50 \mu \mathrm{g})$ per condition were subjected to SDS-PAGE on a $10 \%$ resolving gel and transferred to nitrocellulose membrane (Pall Life Sciences, Mexico). The membranes were blocked with 3\% BSA or 5\% non-fat milk for $1 \mathrm{hr}$ at room temperature followed by incubation with specific primary antibody [CREB (Cell Signaling Technology, \#9197) at 1:1500 dilution, phospho-CREB (Cell Signaling Technology, \#9198) at 1:1500 dilution, HIF-1a (Cell Signaling Technology, \#14179) at 1:1500 dilution, MMP7 (Abcam, ab5706) at 1:1000 dilution, Vinculin (Sigma, V9131) at $1: 10000$ dilution overnight at $4^{\circ} \mathrm{C}$. After washing with $1 \times$ TBST, the membrane was incubated with HRP-conjugated secondary antibody specific for either rabbit (Jackson Immunoresearch Laboratories Inc., 111-035-003 at a dilution of 1:5000 for CREB, phospho-CREB, HIF-1 $\alpha$ and MMP7) or mouse (Jackson Immunoresearch Laboratories Inc. \# 115-035-003 at 1:5000 dilution for Vinculin) for $1 \mathrm{hr}$. The detection was carried out using an enhanced chemiluminescence kit (Bio-Rad, USA) and the luminescence signal was captured using Chemidoc XRS+Chemiluminescence Detection system (Bio-Rad, USA). The intensities of signals were quantified using Image Lab software (Bio-Rad, USA).

\section{Chromatin immunoprecipitation (ChIP) assays}

ChIP assays were carried out as described earlier ${ }^{3}$. Briefly, N2a and H9c2 cells were transfected with MMP7-181G and MMP7-181A promoter-reporter constructs and crosslinkedin 1\% formaldehyde in PBS after $24 \mathrm{hrs}$ of transfection. Chromatin was isolated and sheared by sonication, followed by pre-clearing with Rec protein G-Sepharose 4B beads (Invitrogen, USA). Pre-cleared samples were then immunoprecipitated by incubation with $5 \mu \mathrm{g}$ each of CREB antibody. Non-immune Rabbit IgG (I5006, Sigma) was used as a negative control. The immunoprecipitated chromatin was captured with Rec protein G-Sepharose 4B beads, eluted, reverse cross-linked, digested with RNase 
and proteinase $\mathrm{K}$ and purified using HiPurA ${ }^{\mathrm{TM}}$ PCR product purification kit (HiMedia, India).

Following purification, PCR was carried out to amplify the DNA sequence encompassing the MMP7-181A/G site of polymorphism using the following primers (forward [hMMP7ChIP-230FP], 5'-TGGAGTCAATTTATGCAGCAGACAG-3' and reverse [hMMP7ChIP+22RP], 5'-TTGGACCTATGGTTGATTTGGTG-3') followed by agarose gel electrophoresis of the PCR products. ChIP quantitative PCR was performed using DyNAmoColorFlash SYBR Green kit (Thermo Scientific, USA) using the same primers. The amounts of DNA immunoprecipitated by CREB due to binding to the $-181 \mathrm{G}$ and $-181 \mathrm{~A}$ alleles were quantified by the fold enrichment method relative to lgG signal.

In another set of experiments, N2a cells transfected with MMP7-181G and MMP7-181G promoter-reporter constructs were subjected to treatment with $5.0 \mu \mathrm{M}$ epinephrine or hypoxia treatment followed by isolation of chromatin for ChIP assays. Immunoprecipitation was carried out with $5 \mu \mathrm{g}$ each of CREB and phospho-CREB antibodies (IgG was used as negative control) followed by qPCR of purified immunoprecipitated DNA to quantify fold enrichment relative to lgG.

\section{Building the homology model of CREB1}

Sequence of hCREB1 (UniProtID: P16220) was taken from UniProt (http://www.uniprot.org). Disorder regions and protein binding regions were predicted through DISOPRED3 ${ }^{4}$ server and DNA-binding residues were predicted using DP_Bind ${ }^{5}$ and PredictProtein ${ }^{6}$ server. Then final homology models of human CREB1 (residue 280341) monomer and dimer (with $\mathrm{Mg}^{2+}$ ion), were built through the MODELLER v11.97, using the template PDB-id. 1DH3 (285-339, Mus musculus, mCREB1, resolution: 2.8 $\AA ̊ 丿$ ). hCREB1 and mCREB1 showed $99.7 \%$ sequence conservation. Both have 341 amino acid long CREB1, and out of this 340 residues are conserved except residue at $6^{\text {th }}$ position. Two hundred conformations were generated and the best model with highest negative DOPE_score was selected and further refined and minimized by using OPLS3 force field ${ }^{8}$ through protein-preparation-wizard panel in Maestro v11.2.014 ${ }^{9}$. 
Model's quality assessment was done via Ramachandran plot ${ }^{10}$, ProSA_Zscore ${ }^{11}$, ERRAT ${ }^{12}$, Dope_Score ${ }^{7}$ and secondary structure analysis.

\section{Building of MMP7 promoter DNA models}

Models of MMP7 promoter DNA of 45bp ( $\pm 22 \mathrm{bp}$ flanking -181bp position) for MMP7wild-type with -181A allele and MMP7-mutant with -181G allele were built using the tools 3DDART ${ }^{13}$ and SCFBio (http://www.scfbio-iitd.res.in/software/drugdesign/). Additionally, the DNA models were further refined through Schrodinger of Maestro v11.2.014 by using force field OPLS3 ${ }^{8}$. Furthermore, the 100 ns molecular dynamics (MD) simulation was performed (DESMOND tool of Schrodinger Maestro), to check the stability of DNA in aqueous solution.

\section{Docking of CREB1 to MMP7 promoter DNA}

Protein-DNA docking was performed with $\mathrm{HDOCK}^{14}$ and HADDOCK ${ }^{15}$ tools. In proteinDNA docking with interface information, HDOCK server ranked within the top performed group in CAPRI experiment 2017. HDOCK server utilized hybrid docking algorithm of template-based modeling and free docking. It used hierarchical FFT-based global docking protocol with an improved shape-based pair-wise scoring function to globally sample putative binding modes. HADDOCK generated maximum 200 protein-DNA docking complexes (water-refined models) and clustered them according to their HADDOCK score, more negative score being the more reliable complex. The weighted sum of intermolecular electrostatic (Elec), van der Waals (vdW), desolvation (Dsolv) and ambiguous interaction restraints (AIR) energies and buried surface area (BSA) Term, in total formed HADDOCK score. In HADDOCK server, docking was performed in three successive stages; first, rigid-body docking was performed which generated all possible combination of protein-DNA complex of which top $20 \%$ (based on HADDOCK scores) of the complexes were selected and further refined in semi-flexible refinement stage and finally all filtered complexes were refined in explicit solvent. Z-score of cluster indicates the standard deviation from the average (the more negative the better).

\section{Molecular dynamics simulation of CREB1-MMP7 complexes}

Molecular dynamics (MD) simulation for all CREB-MMP7 (protein-DNA) complexes/models was carried out using Desmond ${ }^{16}$ tool in Maestro. System Builder 
panel was used to build molecular systems. Inbuilt OPLS3 force field was used to assign all parameters. System was solvated with TIP3P 17 water molecules using orthorhombic box with distance of $10 \AA$ from all sides of protein complex. Orthorhombic box volume was minimized by reorientation of the complex/model system. Appropriate number of counter $\mathrm{Na}^{+} / \mathrm{Cl}^{-}$ions was added to electrically neutralized system. The Steepest descent algorithm with 2000 iterations and convergence threshold of 1 $\mathrm{kcal} / \mathrm{mol} / \AA$ was used to minimize all prepared systems, then further equilibration was performed by using default algorithm which include two stage of minimization and 4 stage of MD. Finally, 100 ns of molecular dynamics simulation was performed using NPT ensemble with temperature and pressure coupling of $300 \mathrm{~K}$ and 1 atm respectively. Coordinates and energy were recorded every 10 ps to yield 10000 frames.

MM-GBSA (Molecular Mechanics, the Generalized Born model and Solvent Accessibility) calculation was performed through Schrodinger by using thermal_mmgbsa.py script with step size of 100. A total 100 frames out of 10000 frames were selected for hydrogen bond energy and electrostatic energy calculation. The inbuilt prime MM-GBSA module with OPLS3 force field and generalized Born implicit solvent model in Schrodinger was used to calculate average binding free energy $(\Delta G)$.

\section{Data presentation and statistical analysis}

MMP7 upstream regulatory variants data was collected from Ensemble database. Linkage disequilibrium (LD) analysis was carried out using Haploview $4.2^{18}$. Phenotypic parameters of the study population were expressed as mean \pm S.E. Statistical Package for Social Sciences (SPSS) Version 21.0 was used to carry out genotype-phenotype association studies where the statistical significance of the associations were tested using one-way ANOVA with post hoc tests or Levene's test for equality of variances followed by two-tailed t-test, as appropriate. Transcription factor binding site prediction was carried out using ConSite ${ }^{19}$, MatInspector ${ }^{20}$ and P-Match $^{21}$. Promoter-reporter transient transfections and co-transfections were carried out at least three times and results were expressed as mean $\pm S$.E. of triplicates from representative experiments. Statistical significance was calculated by Student's t-test or one-way ANOVA with Bonferroni's multiple comparisons post-test or two-way ANOVA, as applicable, using 
Prism 5 program (GraphPad Software Inc., USA). All representative graphs were generated using GraphPad Prism 5 software.

\section{SUPPLEMENTARY RESULTS}

\section{Genotyping of MMP7 -181 SNP in Indian populations.}

Genotyping for the MMP7 A-181G SNP was carried out by PCR-restriction fragment length polymorphism (RFLP) method using EcoRI restriction enzyme. Undigested amplicons of $150 \mathrm{bp}$ indicated $-181 \mathrm{~A}$ allele and EcoRI restricted fragment sizes of 120 bp and $30 \mathrm{bp}$ represented the $-181 \mathrm{G}$ allele (Fig. S1). A subset of the samples was subjected to Sanger DNA sequencing (Fig. S1) for confirmation of genotypes. Genotyping results from both these techniques showed $100 \%$ concurrence.

\section{Generation and assessment of the quality of CREB1 model structure.}

While the complete sequence of hCREB1 consists of 341 amino acids (aa) crystal structure of only the 55 aa stretch of CREB1 protein (285-339 aa, PDB ID: 1DH3, species: Mus musculus, identity: $97.7 \%$ ) is available. Therefore, we set out to build a model structure of the transcription factor (TF) hCREB1 for interaction studies with the MMP7 promoter DNA.

First, we carried out in-silico structural profiling of CREB1. Since TFs are known to have long range of intrinsic-disorder zone and undergoes to disorder-to-order transitions upon binding to other partners proteins, ${ }^{22-24}$ we carried out disorderedness analysis on CREB1. DISOPRED3 ${ }^{4}$ was utilized to predict the disordered regions in CREB1. In DISOPRED score, the region with confidence score $>0.5$ was considered as disordered one. The amino acid residues from 1 to 160 were highly disordered (confidence score $>0.8$ ), residue 211 to 280 were moderately disordered (confidence score: $0.6-0.8$ ), and residue 161 to 210,281 to 350 were structured (confidence score < 0.5). This finding is in line with the non-availability of crystal structures for CREB1.

Next, we analyzed the CREB1 sequence to predict DNA binding residues by the DP-Bind ${ }^{5}$ and PredictProtein ${ }^{6}$ tools. The results of DP_Bind and PredictProtein matched 
well with the binding reported in the PDB ID: 1DH3. It was found that the regions of the protein, which mostly interacted with DNA, had stable and fixed secondary structure ${ }^{22}$. From the results of disorderedness prediction and from the DNA-binding, it was clear that only 280-341 region had both properties (viz. fixed secondary structure and DNA binding affinity). Therefore, we generated CREB1 homology model for only this region (280-341).

Homology model of human CREB1 (hCREB1) residues 280-341, was generated through MODELLER v11.910. The final model had $86.8 \%$ residues in the most favored regions in the Ramachandran plot while in the Mus musculus CREB1 crystal structure template 1DH3, 73.6\% residues occurred in most favored region and none of the residues were found in the disallowed region in both the structures. The Prosa Z-score for the modeled CREB1 is -1.87 and -0.97 for CREB template (1DH3). The overall quality factor in ERRAT was 97.8 for the modeled CREB1 and 93.7 for the CREB template 1DH3. The Dope_score and secondary structure predictions for the modeled structure also showed a trend similar to that of the crystal structure (1DH3). The Calpha RMSD analysis (data generated through molecular dynamics simulation) between HD region of model-vs.-1DH3 was $1.1 \AA$, indicating the robustness and good quality of the model.

\section{CREB1 and MMP7-DNA docking predicted greater binding affinity of the mutant promoter.}

Focused protein-DNA docking of MMP7-wild-type and MMP7-mutant (mut) DNA molecules with CREB1 was performed to assess the effect of the A-181G mutation on binding affinity. As shown in the Supplementary Table 4, the CREB1:MMP7-mut complex had relatively higher HADDOCK score (-138.280+/-2.7) and larger cluster size (total complex $=80$ ) as compared to the CREB1:MMP7-wild-type complex score (136.280+/- 3.6) and cluster size (total complex=74) suggesting that MMP7-mut (-181G) DNA may have higher binding affinity as compared to the MMP7-wild-type (-181A) DNA for CREB1. Similar results were also obtained from HDOCK with the best complex having a docking score of -1120.96 and -1110.66, for CREB1:MMP7-mut and CREB1:MMP7-wild-type, respectively. 
Residues forming stable hydrogen bonds with distance up to $3.5 \AA$ and more than $30 \%$ occurrence throughout 100 ns MD trajectory were considered as hot-spot residues. Based on the MD simulations, residues N293 and R301 were identified as hot-spot residues in the CREB1:MMP7-wild-type while in CREB1:MMP7-mut R289 was also identified as a hot-spot residue in addition to N293 and R301.

Of note, structural changes of MMP7-wild-type and MMP7-mut DNA in aqueous system were observed (Figs. S3A and S3B). During MD simulation, the hCREB1(monomer):MMP7-promoter complex also displayed high fluctuations. Therefore, CREB1(dimer):MMP7-wild-type and CREB1(dimer):MMP7-mut complexes were built. From the MD simulations, stable complexes with the lowest RMSD were selected for further analysis (Fig. S3; panels C1, C2 and C3). Both the MMP7-wild-type (average RMSD, $6.9 \AA$ ) and MMP7-mut (average RMSD, $6.4 \AA$ ) DNA evolved simultaneously from 2 to $\sim 7 \mathrm{~ns}$ and then became stable till 100ns. Both the complexes follow the same trend in MD analysis although RMSD calculations suggested that MMP7-mut was more stable than MMP7-wild-type; these results may be attributed to the increased GC content (in the case of the mutant DNA) that improves thermal stability of DNA. Changes in the trajectory of MMP7-wild-type (a shift in RMSD from 2.5 $\AA$ to $6.4 \AA$ ) were observed; however, in case of MMP7-mut, the trajectory remained consistent with value of $2.4 \AA$ throughout 100 ns. Furthermore, a bimodal vs. unimodal distribution was observed in MMP7-wild-type vs MMP7-mut (Fig. S3C1). Chain-wise analysis of CREB1(dimer):MMP7 complex revealed that the changes in RMSD of MMP7-wild-type occurred mainly due chain A where the RMSD distribution showed a significant shift from $3.0 \AA$ to $8.0 \AA$ (bimodal distribution) (Figs. S3C2 and S3C3). In case of MMP7-mut, the RMSD distribution of chain A remained consistent at $3.0 \AA$ throughout the MD simulation and had a unimodal distribution. The Root mean square fluctuation (RMSF) of the Ca atom of the entire residue over different time frame was plotted to measure the fluctuation of the interface residues over the simulation time for MMP7-wild-type and MMP7-mut systems (chain A and B, separately).

We further studied the regions that showed significant structural changes during MD simulations by determining the RMSF difference between MMP7-wild-type and 
MMP7-mut (Fig. S3D). The RMSF difference values for chain B is comparatively smooth and consistent which clearly indicates that the interaction between chain $B$ and DNA is stable in both cases. However, in case of CREB1 chain A:MMP7-wild-type, the RMSF difference had a huge fluctuation when compared to CREB1 chain A:MMP7-mut, validating the outcome of RMSD. The residues that showed large fluctuations in the case of chain A:MMP7-wild-type complex ranged from amino acids 285-292, 305-315 and 334-339. Distance analysis between the MMP7-181 A/G nucleotide and the "center of mass" of residues 285-302 of chain A of CREB1 revealed that the distance was consistent throughout the trajectory with unimodal distribution (mean average distance is $14.0 \AA$ ) in case of MMP7-mut; on the other hand, the distance varied after 40ns and it increased to $25 \AA$ trimodal distribution in case of MMP7-wild-type (Fig. S3E).

Next, we carried out detailed hydrogen bond analysis in MMP7-wild-type and MMP7-mut complexes. While the total numbers of hydrogen bonds were similar in both MMP7-wild-type and MMP7-mut systems up to $20 \mathrm{~ns}$, the number increased in the case of MMP7-mut but not in the case of MMP7-wild-type between $20-35$ ns. Interestingly, after $40 \mathrm{~ns}$ (till $100 \mathrm{~ns}$ ), the total number of hydrogen bonds in the case of MMP7-mut remained consistent and continuous, while no hydrogen bonds were observed in the case of MMP7-wild-type indicating loss of contacts (Fig. 3F). Further, to observe the net thermodynamic changes between MMP7-wild-type and MMP7-mut systems, the MMGBSA energy calculation between CREB1 (chain A) and MMP7 -181 nucleotides A/G was performed. The total electrostatic energy of both systems remained favorable (negative) till $40 \mathrm{~ns}$, however after $40 \mathrm{~ns}$, the energy became positive in the case of MMP7-wild-type, indicating displacement of chain A from the $-181 \mathrm{~A}$ nucleotide (Fig. S3G). Taken together, our extensive computational analysis provided the molecular basis for enhanced interactions of the MMP7 mutant promoter with CREB as compared to the MMP7 wild-type promoter. 


\section{SUPPLEMENTARY TABLES}

Table S1. Clinical characteristics of the Chennai study population.

\begin{tabular}{|c|c|c|c|}
\hline Characteristics & Hypertensive & Control & \\
\hline a) Demographic & Value \pm SD (n) & Value \pm SD (n) & $\mathrm{p}$ value \\
\hline Age (years) & $44 \pm 9(860)$ & $42 \pm 9(638)$ & $<0.01$ \\
\hline $\operatorname{Sex}(M / F)(\%)$ & $70.7 / 29.3(861)$ & $71.1 / 28.9(640)$ & \\
\hline Body Mass Index $\left(\mathrm{kg} / \mathrm{m}^{2}\right)$ & $25.4 \pm 3.6(840)$ & $25.9 \pm 3.6(611)$ & $<0.05$ \\
\hline b) Physiological & Value \pm SEM $(n)$ & Value \pm SEM $(n)$ & $\mathrm{p}$ value \\
\hline Systolic Blood Pressure (mmHg) & $145.7 \pm 0.6(815)$ & $122.3 \pm 0.5(628)$ & $<0.0001$ \\
\hline Diastolic Blood Pressure $(\mathrm{mmHg})$ & $89.7 \pm 0.4(819)$ & $77.9 \pm 0.3(627)$ & $<0.0001$ \\
\hline Mean Arterial Pressure $(\mathrm{mmHg})$ & $108.2 \pm 0.4(815)$ & $92.8 \pm 0.3(627)$ & $<0.0001$ \\
\hline Heart rate & $76.1 \pm 0.3(772)$ & $75.7 \pm 0.3(585)$ & 0.44 \\
\hline c) Biochemical & Value \pm SEM (n) & Value \pm SEM $(n)$ & $p$ value \\
\hline Sodium (meq/L) & $138.9 \pm 0.2(348)$ & $139.5 \pm 0.1(368)$ & $<0.05$ \\
\hline Potassium (meq/L) & $4.28 \pm 0.02(345)$ & $4.32 \pm 0.02(364)$ & 0.15 \\
\hline Urea (mg/dl) & $21.7 \pm 0.2(762)$ & $21.2 \pm 0.2(562)$ & 0.21 \\
\hline Creatinine (mg/dl) & $0.84 \pm 0.01(772)$ & $0.82 \pm 0.01(565)$ & 0.09 \\
\hline Random Blood Sugar (mg/dl) & $111.1 \pm 1.4(752)$ & $104.2 \pm 1.3(550)$ & $<0.001$ \\
\hline Total Cholesterol (mg/dl) & $176.8 \pm 1.4(743)$ & $182.2 \pm 1.5(546)$ & $<0.05$ \\
\hline Triglycerides (mg/dl) & $156.1 \pm 2.9(743)$ & $139.0 \pm 2.8(543)$ & $<0.0001$ \\
\hline $\mathrm{HDL}(\mathrm{mg} / \mathrm{dl})$ & $38.1 \pm 0.3(742)$ & $38.8 \pm 0.3(540)$ & 0.12 \\
\hline LDL (mg/dl) & $109.3 \pm 1.3(747)$ & $115.2 \pm 1.4(551)$ & $<0.01$ \\
\hline
\end{tabular}


Table S2. Clinical characteristics of the Chandigarh study population.

\begin{tabular}{|c|c|c|c|}
\hline Characteristics & Hypertensive & Control & \\
\hline a) Demographic & Value \pm SD (n) & Value \pm SD (n) & $p$ value \\
\hline $\begin{array}{l}\text { Age (years) } \\
\text { Sex }(M / F)(\%)\end{array}$ & $\begin{array}{c}49 \pm 12(493) \\
52.4 / 47.6(494)\end{array}$ & $\begin{array}{c}59 \pm 8(456) \\
70.0 / 30.0(457)\end{array}$ & $<0.0001$ \\
\hline b) Physiological & Value \pm SEM $(n)$ & Value \pm SEM (n) & $\mathrm{p}$ value \\
\hline Systolic Blood Pressure (mmHg) & $147.9 \pm 0.6(492)$ & $118.8 \pm 0.5(441)$ & $<0.0001$ \\
\hline Diastolic Blood Pressure $(\mathrm{mmHg})$ & $95.5 \pm 0.4(508)$ & $75.3 \pm 0.3(442)$ & $<0.0001$ \\
\hline Mean Arterial Pressure $(\mathrm{mmHg})$ & $113.1 \pm 0.4(502)$ & $89.8 \pm 0.3(440)$ & $<0.0001$ \\
\hline c) Biochemical & Value \pm SEM $(n)$ & Value \pm SEM $(n)$ & $p$ value \\
\hline Sodium (meq/L) & $139.6 \pm 0.5(100)$ & $142.8 \pm 0.9(58)$ & $<0.01$ \\
\hline Potassium (meq/L) & $4.70 \pm 0.42(98)$ & $5.03 \pm 0.64(58)$ & 0.65 \\
\hline Urea (mg/dl) & $31.1 \pm 0.9(149)$ & $33.8 \pm 0.9(61)$ & 0.10 \\
\hline Creatinine (mg/dl) & $1.26 \pm 0.24(151)$ & $0.92 \pm 0.04(62)$ & 0.36 \\
\hline Random Blood Sugar (mg/dl) & $94.2 \pm 1.0(97)$ & $96.8 \pm 1.4(58)$ & 0.13 \\
\hline Total Cholesterol (mg/dl) & $190.3 \pm 2.0(454)$ & $185.5 \pm 4.3(65)$ & 0.31 \\
\hline Triglycerides (mg/dl) & $163.6 \pm 2.7(456)$ & $125.7 \pm 4.1(64)$ & $<0.0001$ \\
\hline HDL (mg/dl) & $46.5 \pm 0.5(454)$ & $41.7 \pm 1.1(65)$ & $<0.001$ \\
\hline LDL (mg/dl) & $129.8 \pm 1.9(460)$ & $143.1 \pm 5.0(65)$ & $<0.05$ \\
\hline
\end{tabular}


Table S3. SNPs in the $5 \mathrm{~kb}$ upstream promoter region of MMP7.

\begin{tabular}{|c|c|c|c|c|}
\hline Variant ID & $\begin{array}{c}\text { Chromosome } \\
\text { position }\end{array}$ & $\begin{array}{c}\text { MMP7 } \\
\text { Promoter } \\
\text { Position }\end{array}$ & $\begin{array}{l}\text { Minor Allele } \\
\text { Frequency in Overall } \\
\text { Population }(n=2504)\end{array}$ & $\begin{array}{l}\text { Minor Allele Frequency in } \\
\text { South Asian (SAS) } \\
\text { Population }(\mathbf{n}=\mathbf{4 8 9})^{25}\end{array}$ \\
\hline rs11568819 & 11:102530902 & -153 & 0.040 & 0.047 \\
\hline rs11568818 & 11:102530930 & -181 & 0.358 & 0.443 \\
\hline rs17098318 & $11: 102532127$ & -1378 & 0.301 & 0.370 \\
\hline rs17881620 & $11: 102532522$ & -1773 & 0.255 & 0.328 \\
\hline rs10895308 & $11: 102532844$ & -2095 & 0.209 & 0.083 \\
\hline rs72982143 & $11: 102533418$ & -2669 & 0.038 & 0.044 \\
\hline rs2408391 & $11: 102533760$ & -3011 & 0.238 & 0.270 \\
\hline rs72982145 & $11: 102534227$ & -3478 & 0.042 & 0.053 \\
\hline rs880197 & $11: 102534940$ & -4191 & 0.238 & 0.270 \\
\hline rs1943778 & 11:102535234 & -4485 & 0.031 & 0.039 \\
\hline
\end{tabular}


Table S4.Results of focused protein-DNA docking of CREB1 (monomer) and MMP7promoter DNA through HADDOCK server.

\begin{tabular}{|c|c|c|}
\hline Parameter and score & $\begin{array}{c}\text { CREB1:MMP7-wild- } \\
\text { type }\end{array}$ & CREB1:MMP7-mut \\
\hline HADDOCK score & $-136.274+/-3.6$ & $-138.280+/-2.7$ \\
\hline Cluster size & 74 & 80 \\
\hline $\begin{array}{c}\text { RMSD from the overall lowest- } \\
\text { energy structure }\end{array}$ & $1.3+/-0.9$ & $1.6+/-1.0$ \\
\hline Van der Waals energy & $-49.4+/-2.1$ & $-52.1+/-2.0$ \\
\hline Electrostatic energy & $-632.5+/-19.9$ & $-620.6+/-20.4$ \\
\hline Desolvation energy & $39.5+/-2.5$ & $37.8+/-0.6$ \\
\hline Restraints violation energy & $2.4+/-0.63$ & $2.3+/-0.65$ \\
\hline Buried Surface Area & $1441.3+/-50.6$ & $1452.8+/-39.1$ \\
\hline Z-Score & -1.6 & -1.9 \\
\hline
\end{tabular}




\section{SUPPLEMENTARY FIGURES}

A

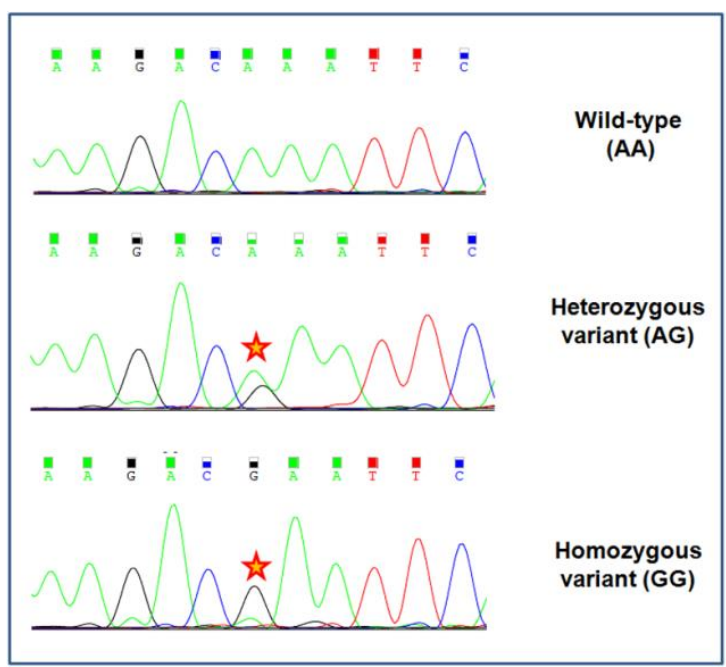

B

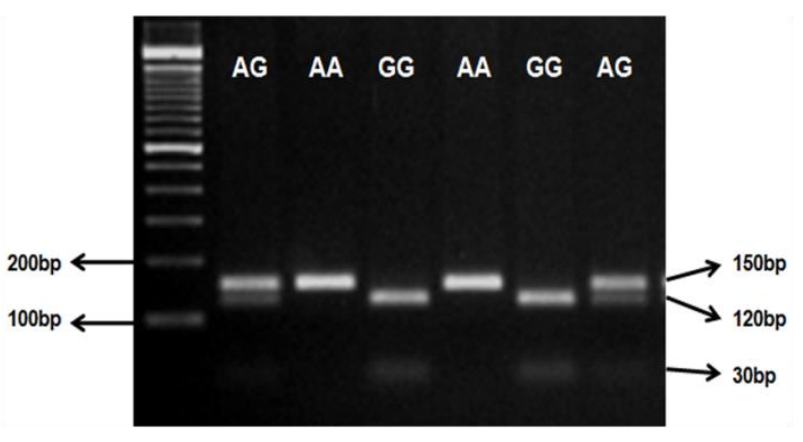

Figure S1. Genotyping of MMP7-181A/G SNP. (A) Chromatograms representing the AA, AG and GG genotypes of MMP7-181A/G SNP (rs11568818). (B) RFLP-genotyping of rs11568818 using EcoRI. The 150 bp PCR product flanking the SNP is cleaved in case of $\mathrm{G}$ allele but not the A allele. Inferred genotypes are indicated. 


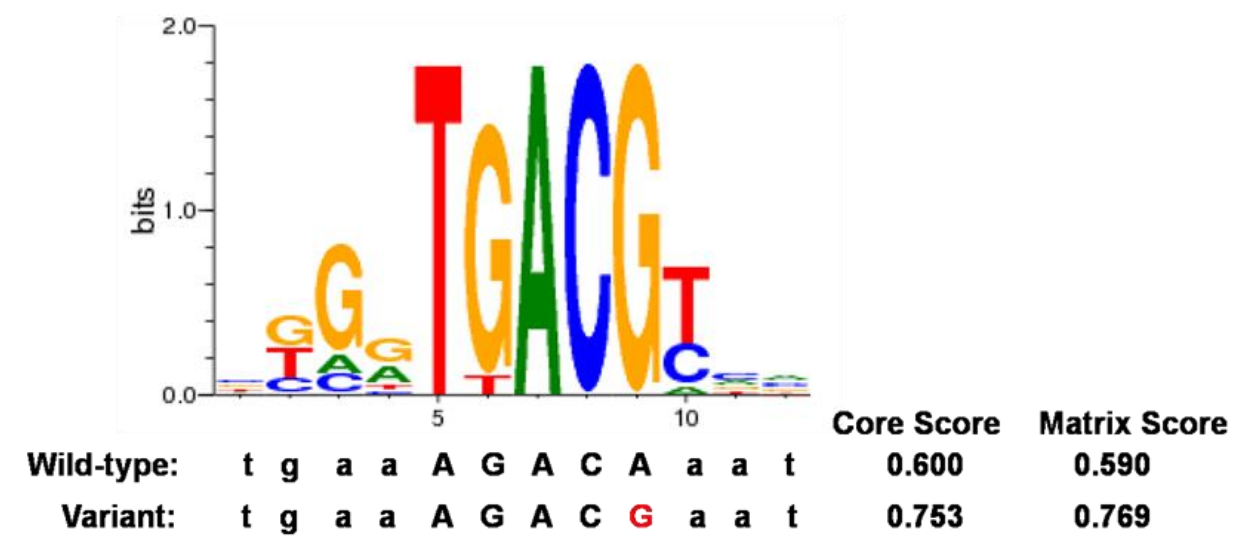

Figure S2. Pictorial representation of matrix for CREB binding motif predicted computationally using P-Match. CREB is predicted to bind to $-181 \mathrm{G}$ allele with higher affinity. 

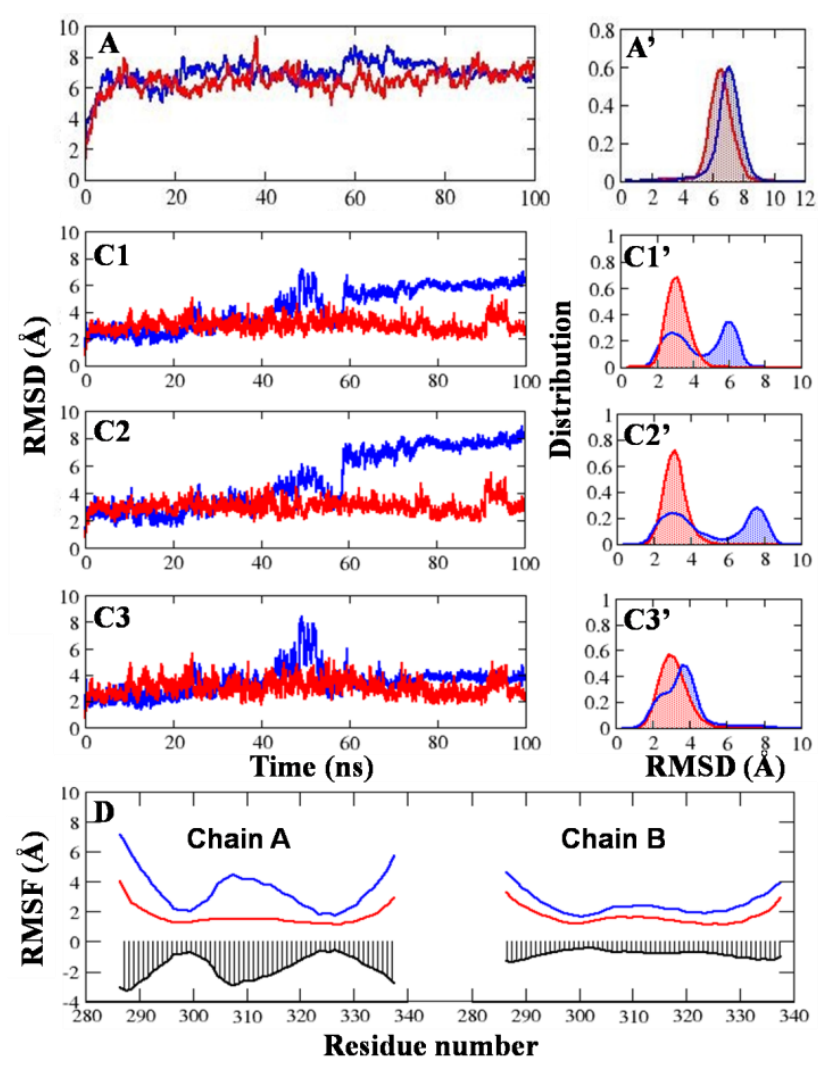
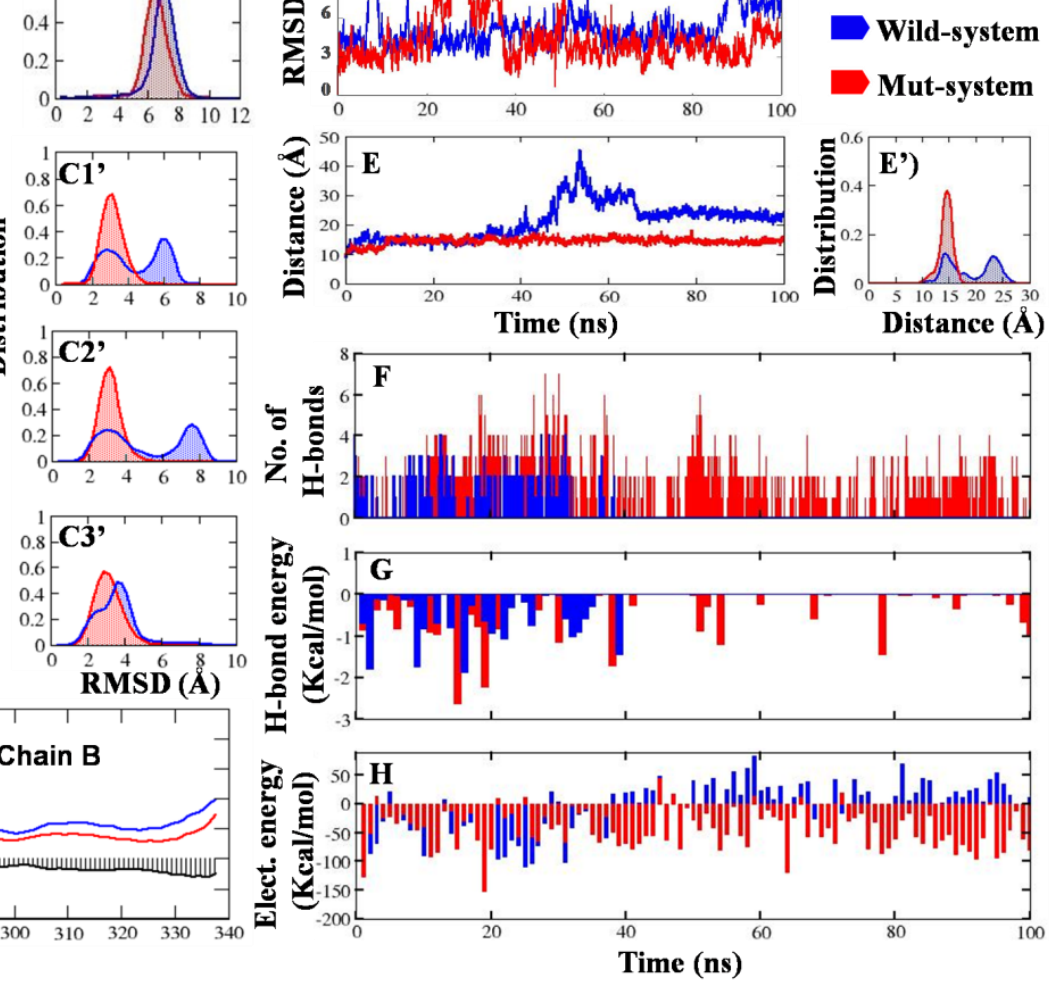

Figure S3. Qualitative and quantitative analysis of wild-type and mut systems: (A and A') RMSD of MMP7-promoter DNA (region \pm 10 bp from $-181 \mathrm{bp}$ ). (B) RMSD of CREB1(monomer)-DNA complex during MD simulations. The CREB1(monomer)-DNAwild-type system showed an average RMSD of $5.0 \AA$ whereas the CREB1(monomer)DNA-mut showed as average RMSD of $4.5 \AA$ AMSD and RMSD distribution profiles from molecular dynamics simulations for CREB1(dimer)-MMP7-promoter complexes: C1 and C1' represent the whole system; $C 2$ and C2' represent chain A; $C 3$ and C3' represent chain B. (D) RMSF profiles of CREB1(dimer)-DNA complexes. (E and E') The distance vs. time evolution and distance vs. distribution profiles between CREB1 chain $A$ and the polymorphic nucleotide (residue number 23) in chain C in CREB1(dimer)DNA complex through 100ns MD simulation. (F, G and $\mathbf{H})$ Hydrogen bonds occupancy, hydrogen bonds energy and electrostatics energy profiles for nucleotide number 23 in chain $C$ and chain A in CREB1(dimer)-DNA complex. 
CREB1:MMP7-Wild

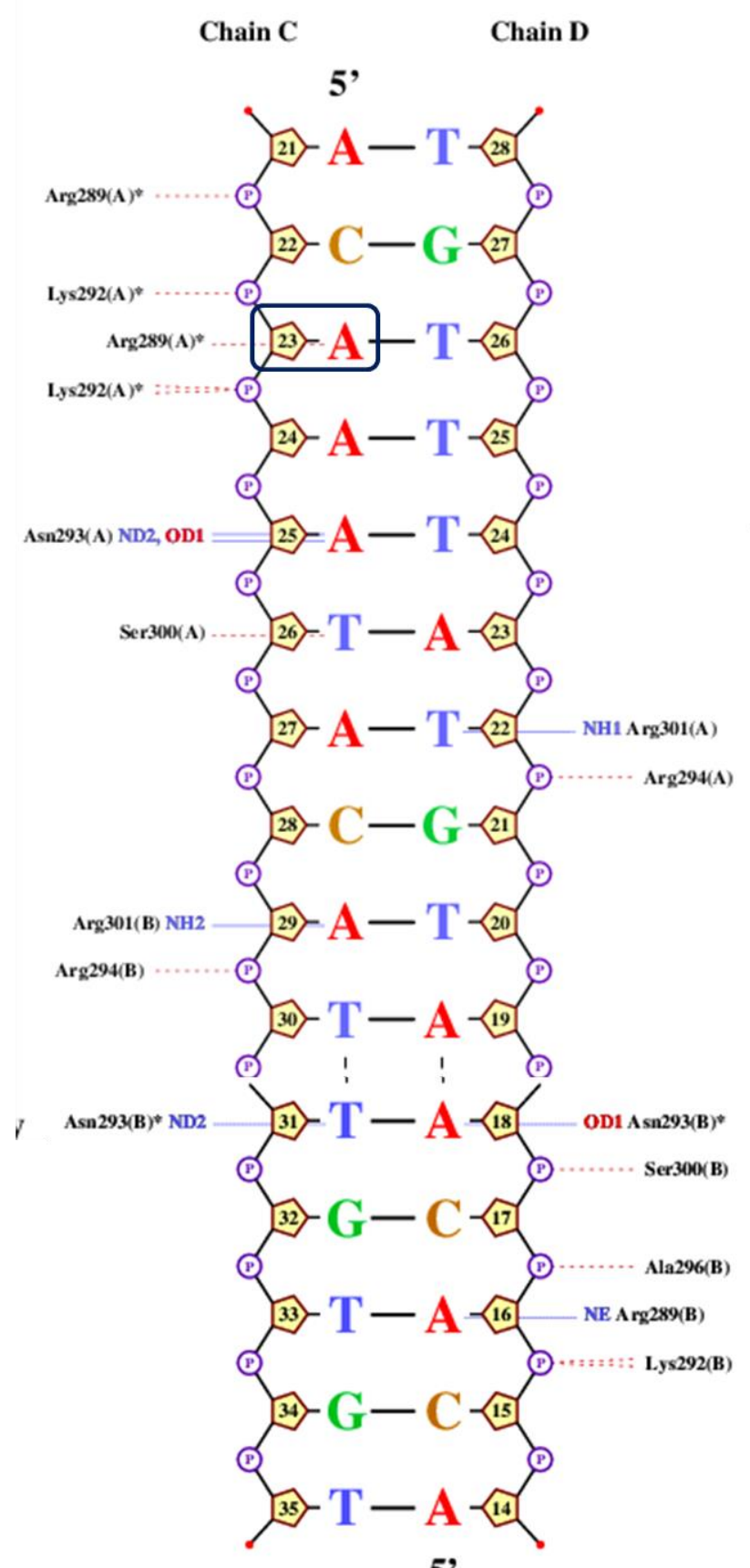

CREB1:MMP7-Mut

Chain C

Chain D

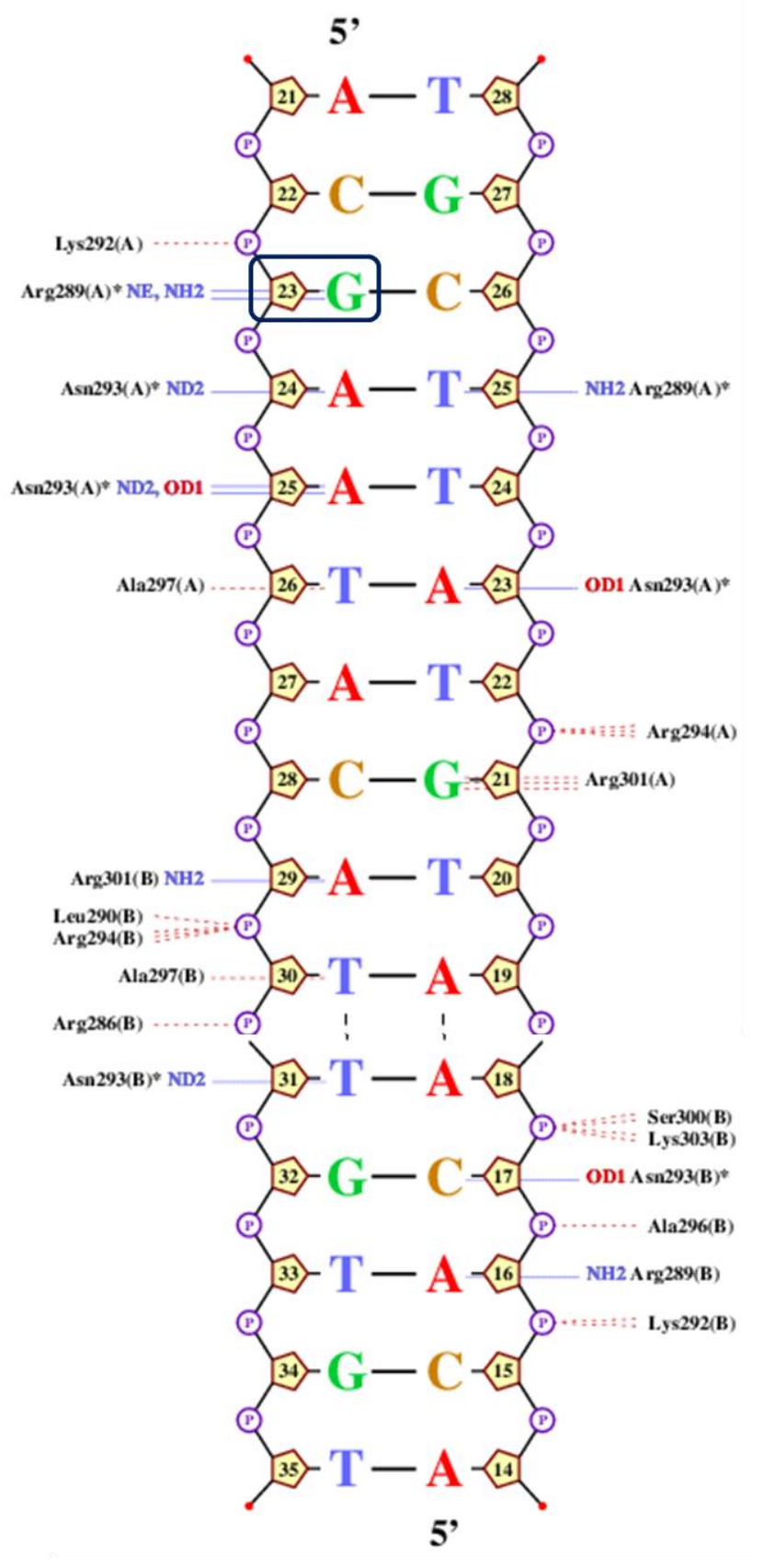

Figure S4. 2D-interaction map of protein-DNA complexes of MMP7-wild-type:CREB1 and MMP7-mutant:CREB1. Hydrogen bonds and van der Waals interactions are represented with solid blue and dotted red lines, respectively. The cut-off distances for hydrogen bonds and van der waals interaction are $3.50 \AA$ and $3.35 \AA$, respectively. This was generated using NucPlot. 
A

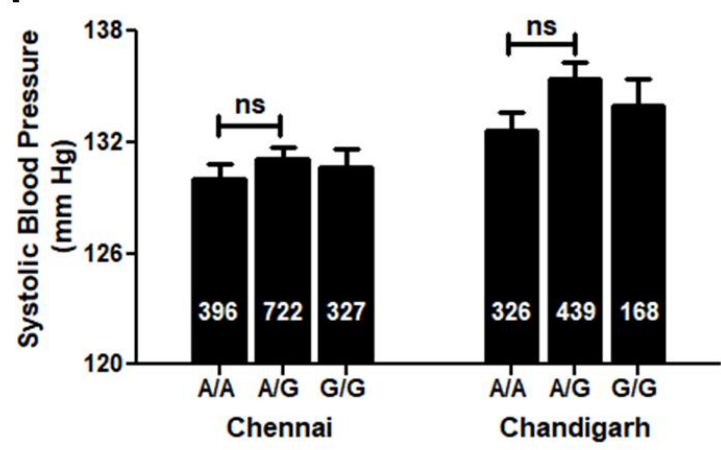

B

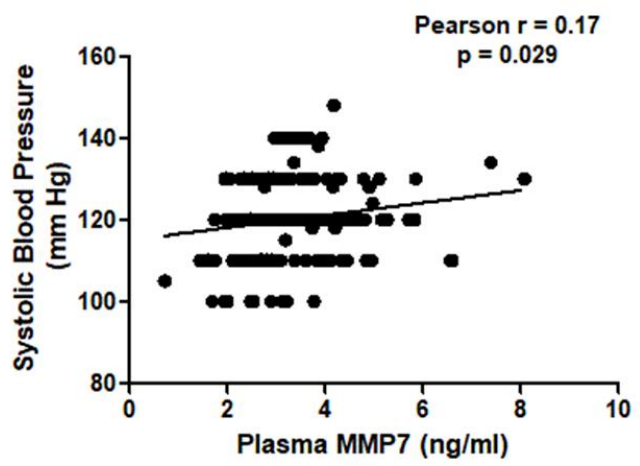

Figure S5. MMP7-181 promoter polymorphism and systolic blood pressure. (A) MMP7$181 \mathrm{~A} / \mathrm{G}$ individuals display a trend towards higher systolic blood pressure in Chennai and Chandigarh populations. (B) Correlation of systolic blood pressure with MMP7 levels. MMP7 levels showed significant positive correlation with systolic blood pressure. Pearson $r$ and $p$ values for the correlations are indicated. 


\section{References}

1. Sonawane PJ, Sahu BS, Sasi BK, Geedi P, Lenka G, Mahapatra NR. Functional promoter polymorphisms govern differential expression of HMG-CoA reductase gene in mouse models of essential hypertension. PLoS One. 2011; 6:e16661

2. Riccio A, Ahn S, Davenport CM, Blendy JA, Ginty DD. Mediation by a CREB family transcription factor of NGF-dependent survival of sympathetic neurons. Science. 1999; 286:2358-2361

3. Kesh K, Subramanian L, Ghosh N, Gupta V, Gupta A, Bhattacharya S, Mahapatra NR, Swarnakar S. Association of MMP7 -181a-->G promoter polymorphism with gastric cancer risk: Influence of nicotine in differential allelespecific transcription via increased phosphorylation of cAMP-response elementbinding protein (CREB). J Biol Chem. 2015; 290:14391-14406

4. Jones DT, Cozzetto D. DISOPRED3: Precise disordered region predictions with annotated protein-binding activity. Bioinformatics. 2015; 31:857-863

5. Hwang S, Gou Z, Kuznetsov IB. DP-bind: A web server for sequence-based prediction of DNA-binding residues in DNA-binding proteins. Bioinformatics. 2007; 23:634-636

6. Rost B, Yachdav G, Liu J. The predictprotein server. Nucleic Acids Res. 2004; 32:W321-326

7. Webb B, Sali A. Protein structure modeling with MODELLER. Methods Mol Biol. 2014; 1137:1-15

8. Harder E, Damm W, Maple J, Wu C, Reboul M, Xiang JY, Wang L, Lupyan D, Dahlgren MK, Knight JL, Kaus JW, Cerutti DS, Krilov G, Jorgensen WL, Abel R, Friesner RA. OPLS3: A force field providing broad coverage of drug-like small molecules and proteins. J Chem Theory Comput. 2016; 12:281-296

9. Small-molecule drug discovery suite 2017-2, schrödinger, LLC, New York, NY,. 2017; 
10. Laskowski RA, Macarthur MW, Moss DS, Thornton JM. \{PROCHECK\}: A program to check the stereochemical quality of protein structures. $J$ Appl Cryst. 1993; 26:283-291

11. Wiederstein M, Sippl MJ. ProSA-web: Interactive web service for the recognition of errors in three-dimensional structures of proteins. Nucleic Acids Res. 2007; 35:W407-410

12. MacArthur MW, Laskowski RA, Thornton JM. Knowledge-based validation of protein structure coordinates derived by X-ray crystallography and NMR spectroscopy. Current Opinion in Structural Biology. 1994; 4:731-737

13. van Dijk M, Bonvin AM. 3d-dart: A DNA structure modelling server. Nucleic Acids Res. 2009; 37:W235-239

14. Yan Y, Zhang D, Zhou P, Li B, Huang SY. HDOCK: A web server for proteinprotein and protein-DNA/RNA docking based on a hybrid strategy. Nucleic Acids Res. 2017; 45:W365-W373

15. van Zundert GCP, Rodrigues J, Trellet M, Schmitz C, Kastritis PL, Karaca E, Melquiond ASJ, van Dijk M, de Vries SJ, Bonvin A. The HADDOCK2.2 web server: User-friendly integrative modeling of biomolecular complexes. J Mol Biol. 2016; 428:720-725

16. Schrödinger release 2017-2: Desmond molecular dynamics system, D. E. Shaw research, New York, NY, 2017. Maestro-desmond interoperability tools, schrödinger, New York, NY 2017;

17. Price DJ, Brooks CL, 3rd. A modified TIP3P water potential for simulation with ewald summation. J Chem Phys. 2004; 121:10096-10103

18. Barrett JC, Fry B, Maller J, Daly MJ. Haploview: Analysis and visualization of LD and haplotype maps. Bioinformatics. 2005; 21:263-265

19. Sandelin A, Wasserman WW, Lenhard B. ConSite: Web-based prediction of regulatory elements using cross-species comparison. Nucleic Acids Res. 2004; 32:W249-252

20. Cartharius K, Frech K, Grote K, Klocke B, Haltmeier M, Klingenhoff A, Frisch M, Bayerlein M, Werner T. MatInspector and beyond: Promoter analysis based on transcription factor binding sites. Bioinformatics. 2005; 21:2933-2942 
21. Chekmenev DS, Haid C, Kel AE. P-Match: Transcription factor binding site search by combining patterns and weight matrices. Nucleic Acids Res. 2005; 33:W432-437

22. Mattapally S, Singh M, Murthy KS, Asthana S, Banerjee SK. Computational modeling suggests impaired interactions between NKX2.5 and GATA4 in individuals carrying a novel pathogenic D16N NKX2.5 mutation. Oncotarget. 2018; 9:13713-13732

23. Bah A, Vernon RM, Siddiqui Z, Krzeminski M, Muhandiram R, Zhao C, Sonenberg N, Kay LE, Forman-Kay JD. Folding of an intrinsically disordered protein by phosphorylation as a regulatory switch. Nature. 2015; 519:106-109

24. Moritsugu K, Terada T, Kidera A. Disorder-to-order transition of an intrinsically disordered region of sortase revealed by multiscale enhanced sampling. $J \mathrm{Am}$ Chem Soc. 2012; 134:7094-7101

25. Genomes Project C, Auton A, Brooks LD, Durbin RM, Garrison EP, Kang HM, Korbel JO, Marchini JL, McCarthy S, McVean GA, Abecasis GR. A global reference for human genetic variation. Nature. 2015; 526:68-74 